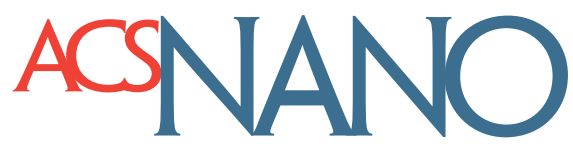

\title{
Coupling Polar Adhesion with Traction, Spring, and Torque Forces Allows High-Speed Helical Migration of the Protozoan Parasite Toxoplasma
}

${ }_{5}$ Georgios Pavlou, Bastien Touquet, Luis Vigetti, Patricia Renesto, Alexandre Bougdour, ${ }_{6}$ Delphine Debarre, Martial Balland, and Isabelle Tardieux*

Cite This: https://dx.doi.org/10.1021/acsnano.0c01893

7 ABSTRACT: Among the eukaryotic cells that navigate through 8 fully developed metazoan tissues, protozoans from the 9 Apicomplexa phylum have evolved motile developmental stages 10 that move much faster than the fastest crawling cells owing to a 11 peculiar substrate-dependent type of motility, known as gliding. 12 Best-studied models are the Plasmodium sporozoite and the 13 Toxoplasma tachyzoite polarized cells for which motility is vital 14 to achieve their developmental programs in the metazoan hosts.

15 The gliding machinery is shared between the two parasites and is

16 largely characterized. Localized beneath the cell surface, it

17 includes actin filaments, unconventional myosin motors housed

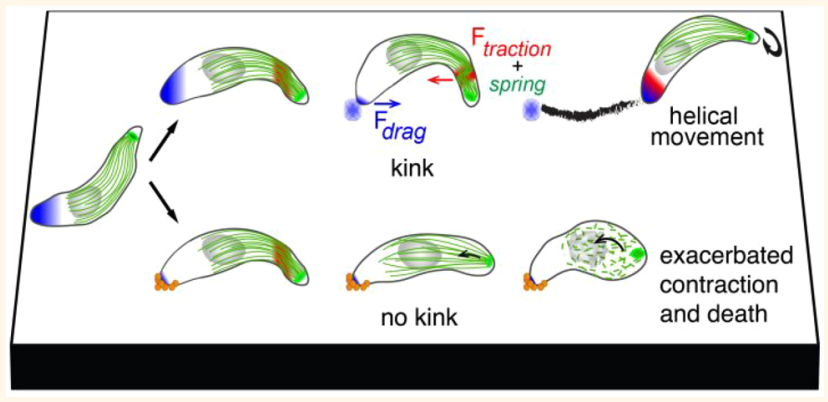

18 within a multimember glideosome unit, and apically secreted transmembrane adhesins. In contrast, less is known about the 19 force mechanisms powering cell movement. Pioneered biophysical studies on the sporozoite and phenotypic analysis of 20 tachyzoite actin-related mutants have added complexity to the general view that force production for parasite forward 21 movement directly results from the myosin-driven rearward motion of the actin-coupled adhesion sites. Here, we have 22 interrogated how forces and substrate adhesion-de-adhesion cycles operate and coordinate to allow the typical left-handed 23 helical gliding mode of the tachyzoite. By combining quantitative traction force and reflection interference microscopy with 24 micropatterning and expansion microscopy, we unveil at the millisecond and nanometer scales the integration of a critical 25 apical anchoring adhesion with specific traction and spring-like forces. We propose that the acto-myoA motor directs the 26 traction force which allows transient energy storage by the microtubule cytoskeleton and therefore sets the thrust force 27 required for $T$. gondii tachyzoite vital helical gliding capacity.

28 KEYWORDS: cell migration, cell focal contact, 3D collagen, Toxoplasma, traction force microscopy,

29 reflection interference contrast microscopy, expansion microscopy

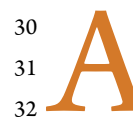

cross eukaryotic phyla, cell migration is an evolutionary conserved function which appears vital for both free living protozoans and metazoans. ${ }^{1}$ In metazoans, cells 33 such as fibroblasts and leukocytes use a wide spectrum of 34 adhesion-dependent motility modes conjointly defined as 35 crawling and primarily driven by the dynamics of the cell 36 actin-myosin cytoskeleton. ${ }^{2,3}$ These cells crawl with the help 37 of dynamic actin-powered membrane protrusions that fold at 38 the leading edge as a flat lamellipodium or as a variety of 39 pseudopodia-like membrane projections. Firmly attached to 40 the substrate upon coordinated assembly of integrin-driven 41 adhesive platforms, the lamellipodium is a hallmark of the 42 mesenchymal cell migration mode in $2 \mathrm{D}$ and $3 \mathrm{D}$ micro- environments. These anchoring multimolecular platforms 43 allow the development of actomyosin bundles that tune 44 contractile forces between the front and trailing margins and 45 eventually lead to the release of trailing adhesions, hence 46 allowing cell forward translocation., ${ }^{4,5}$ Likewise, metazoan 47 cells-as well as large free-living protozoans-can display a 48

Received: March 4, 2020

Accepted: May 20, 2020

Published: May 20, 2020 
49 more versatile motility mode and cope with heterogeneous 3D 50 microenvironments. In addition to the lamellipodium style, 51 they can adopt diversified pseudopodia mechanisms that, 52 combined with a range of relative cell-matrix adhesion and 53 actomyosin contractility, account for the rapid amoeboid type 54 of motility ( $\sim 10$ to $20 \mu \mathrm{m} / \mathrm{min}$ versus less than $1 \mu \mathrm{m} / \mathrm{min}$ for 55 the lamellipodia mode). ${ }^{6}$ Alternatively, fibroblasts can utilize 56 an amoeboid-mesenchymal hybrid type of 3D migration 57 called lopobodial during which a pressure-driven bleb-like 58 membrane protrusion stems at the leading margin from the 59 piston-like forward movement of the nucleus itself driven by 60 the cortical actomyosin contractility. ${ }^{7}$ Biophysical studies have 61 proved instrumental in mapping and measuring the develop62 ment of forces in cells, and they brought insights on how cells 63 differentially regulate the adhesion-contractile balance to 64 spatiotemporally control the adhesion assembly deassembly 65 cycle and achieve productive forward movement in different 66 contexts. $^{8}$

67 Intriguingly, unicellular eukaryotic parasites from the ancient 68 phylum of Apicomplexa have evolved an adhesion- and actin69 dependent motility mode that differs from the mesenchymal, 70 amoeboid, lobopodial repertoire and enables the microbes to 71 glide within host tissues and across barriers to follow their 72 complex stepwise developmental program. The several 73 thousand members of the Apicomplexa phylum include 74 among the world's most pre-eminent mammal-invasive 75 protozoan parasites. In humans, Plasmodium spp. are 76 inoculated in the vascularized dermis extracellular matrix 77 (ECM) by a blood-feeding mosquito which hosts parasites in 78 salivary glands. Plasmodium displays a prolonged asexual 79 developmental phase in red blood cells, which results in a 80 range of clinical outcomes known as malaria, impacting human 81 populations in tropical and subtropical regions of the Earth. Its 82 relative Toxoplasma gondii is present in a large assortment of 83 endotherm metazoans worldwide. Being ingested with 84 contaminated food and water, T. gondii is hosted quasi-silently 85 by possibly up to a third of the human population in tissues 86 such as the brain, retina, and heart and skeletal muscles. 87 However, in case of immune dysfunction, T. gondii can re88 emerge from these tissues and proliferate, causing life89 threatening or debilitating complications. ${ }^{9}$

90 Throughout their life cycle in their respective hosts, both 91 Plasmodium and T. gondii ensure their perpetuation by 92 undergoing multiple cell differentiation events that give rise 93 to specific developmental stages with sizes of several microns, 94 harboring a crescent shape and endowed with high motility 95 skills. ${ }^{10}$ Best studied are the Plasmodium slender mature 96 sporozoite inoculated in the host skin and the T. gondii bulky 97 highly replicative tachyzoite stages that glide 10 times faster 98 than the amoeboid cells without folding any of the crawling99 associated protrusions. ${ }^{10-12}$ Instead, they share a robust 100 longitudinal apico-basal polarity which persists during the 101 generally unidirectional motile activity with the apex leading. ${ }^{13}$ 102 Whereas the Plasmodium sporozoite moves on a circular path 103 in either counterclockwise (CCW) or clockwise (CW) 104 directions with an irregular stop and go kinetics, ${ }^{13}$ it can also 105 occasionally adopt a random motion or a corkscrew-like 106 pattern in vivo. ${ }^{14,15}$ Instead a left-handed helix characterizes the 107 path the mosquito-restricted ookinete Plasmodium stage used 108 to move in Matrigel-based 3D matrices. ${ }^{16}$ The Toxoplasma 109 tachyzoite can similarly glide CCW on a circular path under $1102 \mathrm{D}$ in vitro settings but often uses a CCW helical trajectory or a 111 succession of the two mechanisms over the same gliding sequence. $^{11,17}$ Another intermittent 2D movement termed 112 twirling accounts for the stationary $\mathrm{CW}$ rotation executed by 113 the upright-positioned tachyzoite around its posterior end. ${ }^{18} 114$ The corkscrew-like mode of gliding, which is the obligate 115 mechanism in 3D reconstituted or in vivo environments was 116 proposed to relate to the $2 \mathrm{D}$ helical mode of gliding. ${ }^{19}$ The 117 latter typically starts with a CW short motion, follows with a 118 $180^{\circ}$ twist; meanwhile, the body flattens on the substrate 119 before twisting up and flipping on the side, which allows 120 starting another helical cycle. ${ }^{11,18}$

To initiate and sustain all types of gliding activity, both the 122 sporozoite and tachyzoite rely on the regulated secretion of 123 adhesive protein oligomers at the cell surface ${ }^{20,21}$ that, once 124 delivered at the apical tip, can coengage with ECM proteins 125 and short cortical actin filaments organized beneath the plasma 126 membrane. Force production driving parasite movement has 127 long been considered to directly translate from the apico-basal 128 translocation of the ECM-bound adhesins coupled to the 129 flowing actin filaments and their enzymatic-mediated release of 130 these adhesins at the trailing edge. ${ }^{22,23}$ It is the Apicomplexa 131 specific, fast, nonprocessive, single-headed motor myosin A 132 (MyoA) that was assigned an essential powering role in pulling 133 backward the apically initiated actin filaments and the captured 134 adhesins. $^{24,25}$ Upstream from MyoA however, the T. gondii 135 tachyzoite additionally requires myosin $\mathrm{H}(\mathrm{MyoH})$ to propel 136 actin filaments from the parasite apical tip formed by the 137 conoid, $^{26}$ a retractile appendage missing in Plasmodium. Both 138 $\mathrm{MyoA}$ and $\mathrm{MyoH}$ molecules function within a multisubunit 139 complex identified as glideosome ${ }^{22}$ that also comprises several 140 regulatory subunits and scaffolding partners. ${ }^{27-29}$ Specifically 141 for MyoA, the glideosome associated proteins (GAPs) anchor 142 the motor machinery to the plasma membrane and the lining 143 internal bilayered membrane complex (IMC) which underside 144 is tethered to a cytoplasmic array of cortical microtubules 145 (cMTs), the number and arrangement of which typify each 146 Apicomplexa species and developmental stage. ${ }^{27,30} \quad 147$

However, the recent introduction of force microscopy, 148 reflection interference contrast microscopy (RICM) and 149 optical tweezers in conjunction with molecular genetics in 150 the Plasmodium field has added more complexity to the 151 prevalent force production model. ${ }^{31}$ A key observation is the 152 discrepancy between the speed of the retrograde flow of 153 optically trapped microbeads on the apical surface of the 154 sporozoite and the speed achieved during typical circular 155 gliding, the former being up to $50 \%$ faster than the latter. ${ }^{31}$ In 156 addition, several distinct areas of contact between the moving 157 sporozoite and the substrate were uncovered at the apex, base, 158 and center over the circular path and were shown to display 159 specific on-off dynamics, ${ }^{13}$ whereas the genetic loss of a 160 surface-exposed adhesin reported to function during motility 161 was found to trigger a speed increase in the retrograde flow and 162 a concomitant decrease in force production. ${ }^{31}$ Collectively, 163 these studies bring compelling evidence for the assembly of 164 distinct force-generating adhesive structures which differ- 165 entially control production of the force required for migration. 166

By contrast, how forces and dynamic adhesions coordinate 167 in space and time and integrate with the impressive high-speed 168 helical path that features the T. gondii tachyzoite gliding 169 mechanism has been much less investigated. Intriguingly, the 170 rearward capping of nanobeads bound to the tachyzoite surface 171 was shown not to require an intact actomyosin system but 172 rather a polarized secretory-endocytic cycle that would locally 173 drive membrane tension. ${ }^{32}$ On the other hand, qualitative 174 
A

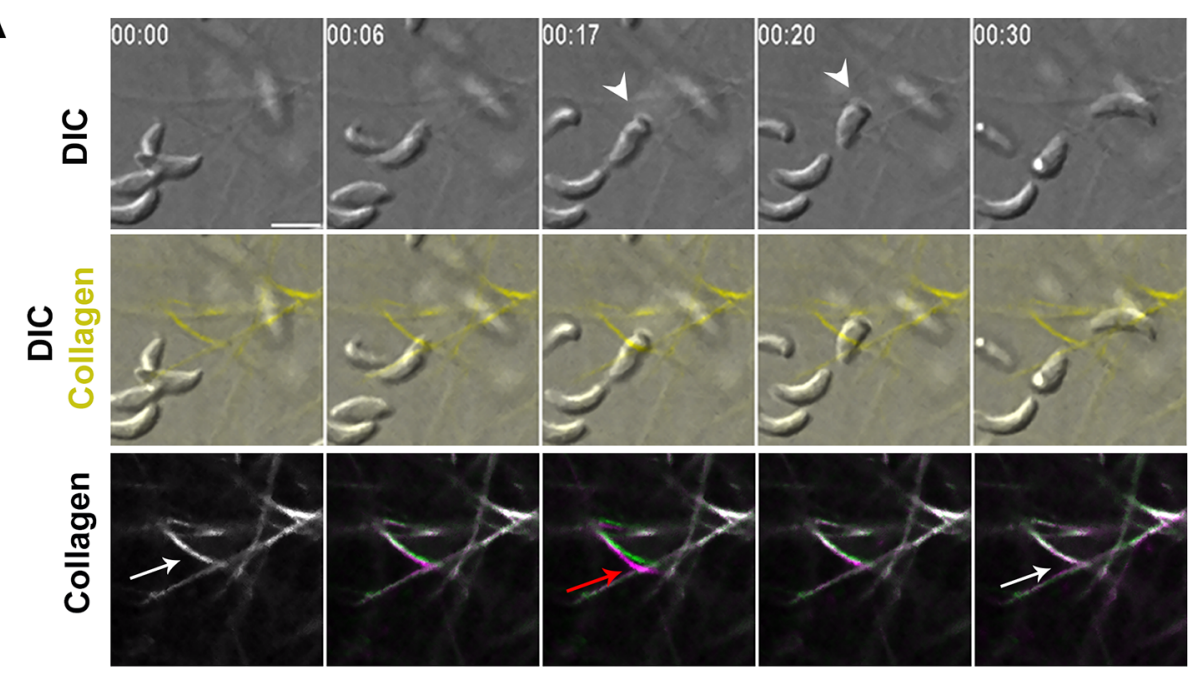

B
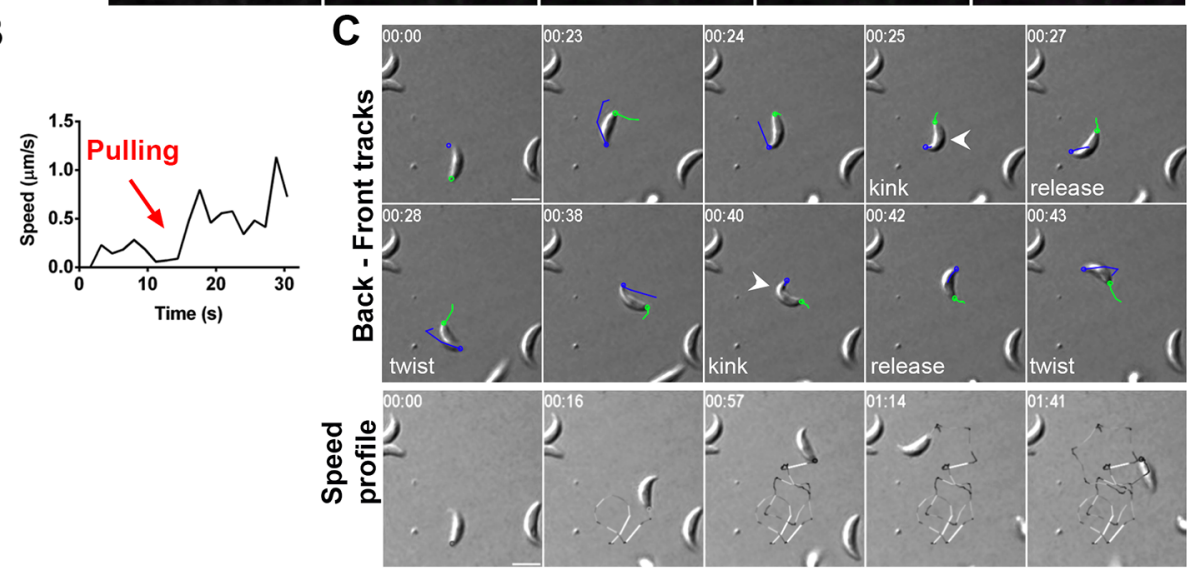

D
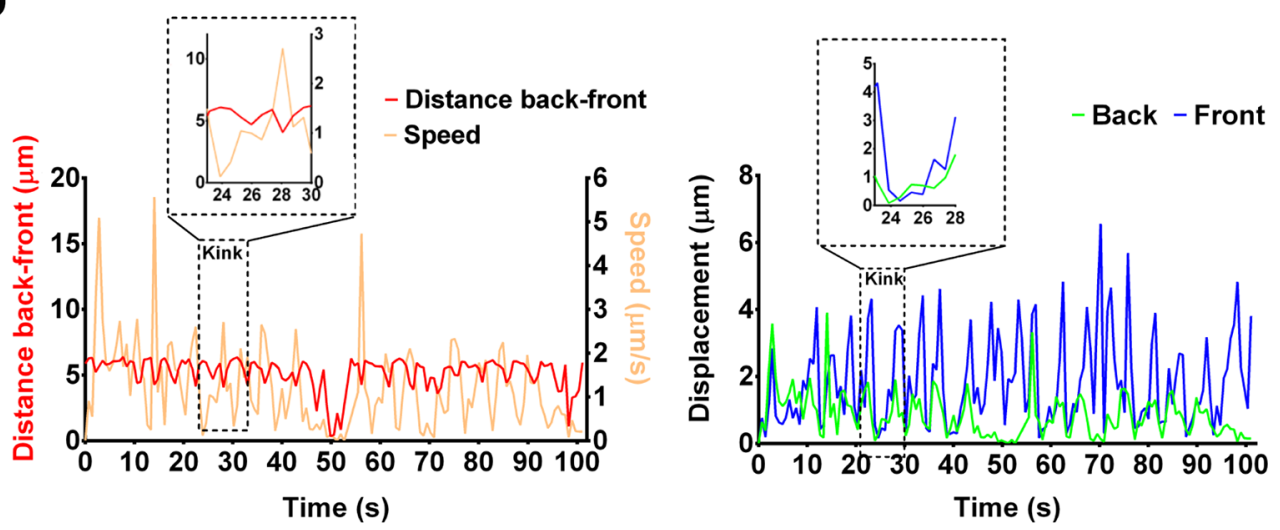

Figure 1. Tachyzoite pulls on the collagen fibers prior to helical acceleration. (A) Successive images from a time lapse of a tachyzoite navigating within a 3D-meshwork of rat collagen 1 fibers prelabeled with the CNA35-GFP collagen chimeric probe. Panels show images of (top) DIC, (middle) DIC-GFP over, giving yellow-colored fibers, (bottom) the fiber initial ( $t_{0}$, green) and over time $\left(t_{x}\right.$, magenta) positions when encountered by the moving tachyzoite. The overimposition of the green and magenta signals comes as white (white arrows) when the fiber maintains the $t_{0}$ position, whereas the separation between the green $\left(t_{0}\right)$ and magenta $\left(t_{x}\right)$ signals attests two distinct positions overtime. The backward displacement indicates the parasite pulls on the fiber (red arrow), whereas the graph (B) documents that this pulling coincides with the parasite low speed. Next the acceleration step coincides with the merge back of the green and magenta signals that signs for the release of fiber tension post motion. (C) Successive DIC images from a time lapse of a tachyzoite undergoing helical gliding on fibronectin-coated glass. (Top) back (green) and front (blue) tracks over three last frames $\left(t_{x-2}\right.$ to $\left.t_{x}\right)$ are shown, (bottom) speed profile is shown from black to white scaling with increasing speeds. All white arrowheads point to the kink, all scale bars: $5 \mu \mathrm{m}$, time is in minutes/ seconds. (D) Graph (left) plots the tachyzoite back-front distance (red line) and the speed based on the back tracking (light orange); note that the value close to zero corresponds to the time the tachyzoite twirls, hence the apex is out of focus and the back-front distance not measurable (right) the $x y$ displacement for both the back (green) and front (blue) sites over the helical sequence. Zoomed of a typical kink step for both graphs shown as inlays.

175 RICM allowed identifying an early tachyzoite contact with the 176 substrate at the cell front and its rearward translocation which was assumed to directly power the force-producing parasite 177 movement. $^{33}$ In this study, we revisited the mechanisms 178 
A

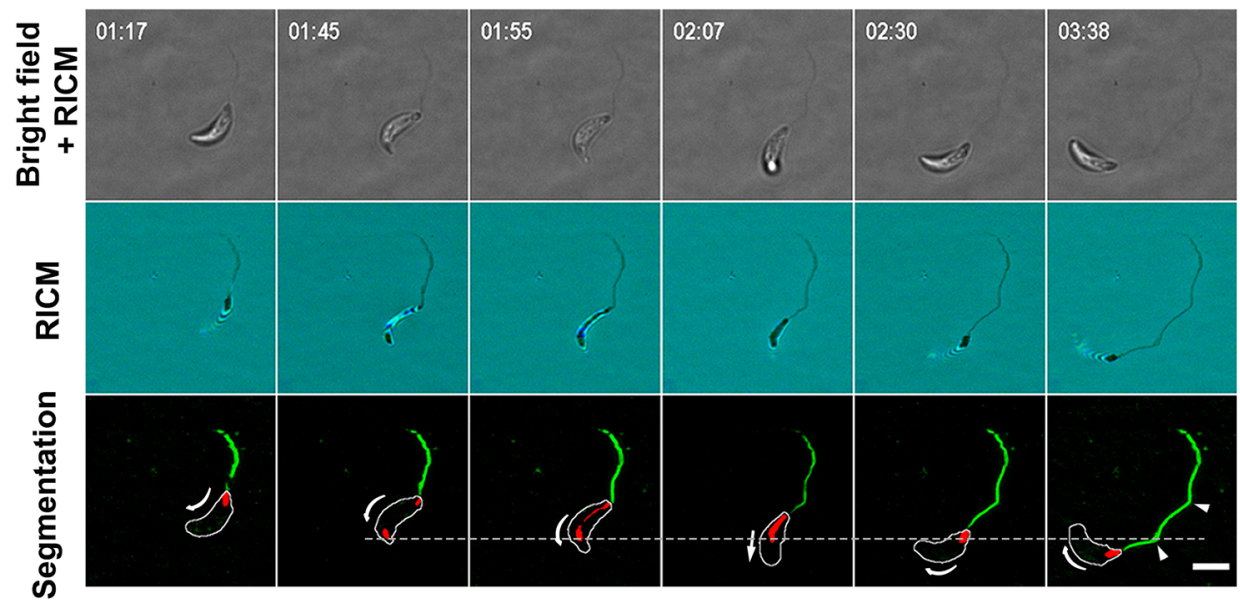

B

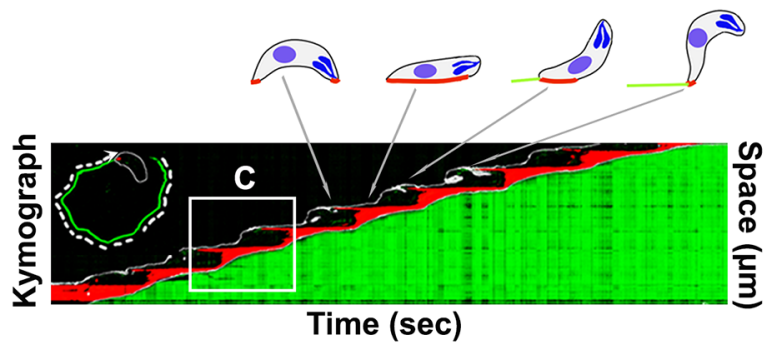

C

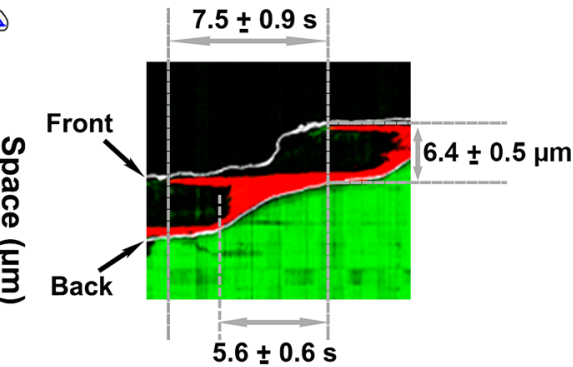

D

E
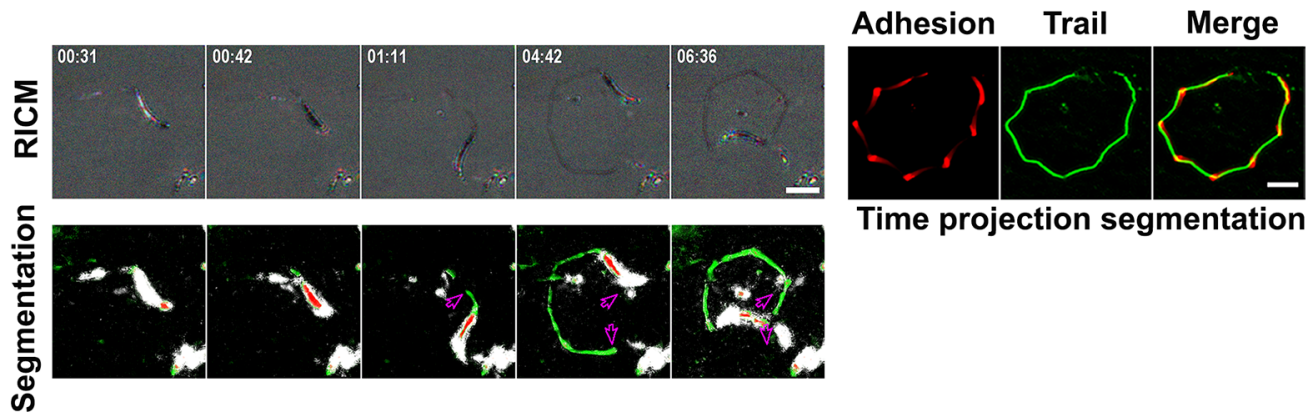

Time projection segmentation

Figure 2. Helical cycle starts with an apical site of adhesion that constraints sliding of both the parasite and the parasite posteriorly shed trail. (A) Successive RICM images from a time lapse of a tachyzoite undergoing helical motion on a glass coverslip. Panels show (top) simultaneous bright-field (BF) and RICM images, (middle) blue and green RICM images superimposed and displayed as RGB, (bottom) segmentation of BF and RICM images showing the parasite outline (white), areas of close contact between the glass and the moving parasite (red), and material shed behind as trail (green). White arrows indicate the direction of the parasite movement. The dotted line marks the position of the initial contact over one helical cycle and delineates the trajectory angular point. (B,C) Kymograph of the whole gliding sequence from (A) and for a single zoomed helical cycle (C) shows the evolution of the close contact area along the trajectory over time. (D) Successive three-color RICM images (shown as RGB) from a time lapse of a tachyzoite undergoing helical motion on a poly-L-lysine-coated glass coverslip (top) and corresponding segmentation with the Weka FIJI plugin (bottom). The pink arrows indicate that over time the tachyzoite pulls on the trail that slides without breaking but follows the polygonal trajectory imposed by the initial anchoring sites. (E) Time projection of the segmented close contact areas over the gliding sequence on which (left) the longest-lived attachment points are brightest, (middle) the trails are green, and (right) adhesion and trails are overimposed. Note that angles in the polygonal path coincide with both the long-lived close contacts and the kinks. All scale bars: $5 \mu \mathrm{m}$; time is in minutes/seconds.

179 underpinning the helical gliding of $T$. gondii by combining 180 quantitative live imaging with traction force microscopy, 181 quantitative RICM, micropatterning and expansion micros182 copy. We provided evidence for a spatiotemporal coordination 183 of an actoMyoA-based traction force produced at a stable 184 apical adhesion site and a spring-like force which, driven by the left-handed cMT spiral orientation, directs straightening and 185 helical propelling of the $T$. gondii tachyzoite.

\section{RESULTS/DISCUSSION}

Tachyzoite Pulls on the Collagen Fibers in a 3D 188 Meshwork Prior to Accelerating with a Helical Motion. 189 
190 Pioneering work on $T$. gondii 3D in vitro motility has relied on 191 Matrigel-based matrices under rather elastic and homogeneous 192 conditions. ${ }^{19}$ However, in vivo, most ECM microenvironments 193 are enriched in a nonlinear, anisotropic, and dynamic fibril-rich 194 meshwork. Because collagens are dominant ECM components 195 that form multiscale fibrils withstanding tensile forces, ${ }^{34}$ we 196 analyzed how the $T$. gondii tachyzoite moves within a confined 197 collagen fibril-based matrix. Whereas the tachyzoite squeezed 198 its body to pass through the meshwork, it first paused and 199 bent, showing an apical deviation as it next accelerated, 200 possibly with a rotation around its main axis, to escape from 201 the collagen fibrils (Figure 1A,B and Supporting Information 202 Figure S1, Movie 1, and Movie 2). Using a fluorescent probe 203 selectively associated with collagen fibrils, ${ }^{35}$ we applied a 204 temporal color-code (green and magenta) and identified fibers 205 encountered by the motile tachyzoite that shifted backward 206 before returning to initial position as the parasite propelled 207 forward (Figure 1A, Supporting Information Movie 1). This 208 fiber backward displacement was seen in all cases of moving 209 tachyzoites encountering a fiber $(\mathrm{n}=10)$, and qualitatively 210 indicated that the parasite exerted pulling forces on the 211 surrounding fibers.

212 To get more mechanistic insights on the front-rear 213 spatiotemporal coordination over the helical gliding cycle, we 214 further analyzed the changes in the tachyzoite shape with high 215 spatiotemporal acuity using a $2 \mathrm{D}$ setting. We observed a yet 216 undefined apical inward bending immediately post the initial 217 clockwise arc motion and concomitant with the parasite pause 218 (Figure 1C). Indeed, tracking the front and back revealed a 219 shortening of the parasite that coincided with an apical flexure 220 and thus an increased curvature, resembling the observation 221 made within the fibrous 3D matrix, and which we defined as 222 the kink step. Associating with the apical pole arrest we 223 detected the contraction of the cell body and retraction of the 224 posterior pole. Next the apex re-extended and lifted up, a phase 225 defined as the kink release, while the tachyzoite straightened 226 and slid to further twisted in the typical CCW motion (Figure 227 1C, Supporting Information Movie 3a and Movie 3b, slow 228 motion). Accordingly, not only the instantaneous speed was 229 about null during the kink step and reached maximal value as 230 the latter relaxed back (Figure 1D), resulting in the 231 characteristic periodic speed fluctuations documented under 232 3D conditions. ${ }^{19}$ No such pattern was detected when the 233 parasite performed the 2D-restricted CCW circular trajectory 234 (Supporting Information Figure S1B,C and Movie 4). Of note, 235 high temporal resolution live imaging of Plasmodium 236 sporozoites also reveals alternating periods of rapid movement 237 in which they travel up to one body length and periods of slow 238 movement in which they more firmly adhere to the substrate, 239 thereby indicating that distinct adhesion types operate during 240 motility. ${ }^{13}$ Although such kinetics was associated with the 241 sporozoite body stretching upon firm adhesion of its polar and 242 center regions to the substrate and the subsequent thrust in 243 speed, ${ }^{13}$ no peculiar apical deformation that could resemble a 244 kink has been observed to initiate movement. We proposed 245 that the kink deformation and the subsequent body extension 246 might reflect the tension built in the tachyzoite cytoskeleton to 247 achieve the thrust force required for the helical twisting 248 motion.

249 Apical Focal Adhesion Behaves as a Firm Long-Lived 250 Anchor That Constrains Sliding of the Parasite Body. 251 Because the apical kink suggests the assembly of a specific 252 contact between the parasite front and the substrate, which would be appropriate for building up tension in the T. gondii 253 cytoskeleton, we first used qRICM to monitor the parasite- 254 substrate distance. In contrast to a recent study, ${ }^{33}$ we used 255 high-speed (30 frames/s), multicolor RICM with simultaneous 256 transmitted-light imaging coupled with machine-learning-based 257 image processing to achieve a precise segmentation of close 258 contact regions between the parasite and the substrate at high 259 temporal resolution $(33 \mathrm{~ms}$ ) over thousands of frames (Figure $260 \mathrm{f} 2$ 2 and Supporting Information Movie 5 and Movie 6). In $261 \mathrm{f} 2$ addition to the low-distance patches detected between the 262 parasite basal pole and the substrate even in absence of 263 movement, we found that at the onset of a helical cycle, the 264 membrane of the tachyzoite apex came in contact with the 265 glass surface. Because these RICM-detected signals coexisted 266 with motion, we assumed they accounted for two polar 267 parasite-substrate adhesive areas in agreement with Tosetti 268 and collaborators ${ }^{33}$ and similarly to what was found for the 269 Plasmodium sporozoite. ${ }^{13}$ Crucially, the apical contact 270 remained static over the full length of the helical cycle ( 7.5271 $\pm 0.9 \mathrm{~s}$ ), and the posterior contact progressively expanded to 272 account for $\sim 80 \%$ of the tachyzoite length along its major axis 273 (Figure 2A-C, see kymographs, Supporting Information 274 Movie 5). Upon release of the kink, the parasite propelled 275 forward and shifted orientation before it started a new front 276 attachment. For each helical cycle, the kink step could be a 277 posteriori inferred through the mix of parasite-derived 278 membrane hydrophobic droplets and surface-exposed pro- 279 teins/glycoproteins left as a complex trail behind the cell edge 280 onto the substrate, a common feature of Apicomplexa motile 281 stages (Figure 2A,D,E). ${ }^{11,36}$ Quantitative modeling of the 282 RICM signal provides transverse dimensions for these trails of 283 $\sim 70 \mathrm{~nm}$ (isotropic) or $100 \mathrm{~nm}$ (width) and 40-60 nm 284 (height). By comonitoring the parasite helical motion and the 285 trail assembly in real time, we were able to uncover that the 286 tachyzoite not only slid on the adhesive tracks it built itself but 287 could also pull these, an observation that has not been reported 288 for the RICM data collected on Plasmodium. ${ }^{13}$ These 289 observations suggest a continuous nature of the shed material 290 that remained close but not firmly fixed on the substrate 291 (Figure 2D and Supporting Information Movie 6), which 292 would agree with membrane components including lipids as 293 previously deduced from the analysis of fixed samples. ${ }^{11} 294$ Importantly, the sliding trajectories of the shed material once 295 pulled by the moving tachyzoite appeared constraint by the 296 periodically distributed firm anchors, which could occasionally 297 be stripped off from the substrate (Supporting Information 298 Movie 6). In further support of these periodic stable anchors, 299 the time projection of the trail trajectory postimage 300 segmentation allowed positioning the longest-lived parasite- 301 substrate contacts at each angular point of the trajectory, 302 matching the initial apical anchoring sites (Figure 2E). The 303 relative homogeneity in the trail observed at our RICM 304 resolution cannot inform on a specific distribution or 305 arrangement in proteins and lipids upon specific secretory 306 events or/and due to local physical constraints that would 307 compose the periodic anchors. The microneme-stored MIC2 is 308 seen as the adhesion prototype that bridges the parasite motor 309 system and ECM ligands during motility. Indeed partial or full 310 silencing of the mic 2 expression significantly impairs both the 311 helical and twirling motions. ${ }^{37,38}$ This double default argues 312 more for a MIC2 contribution to the posterior adhesion 313 force, ${ }^{37}$ hence mic2 $\mathrm{KO}$ parasites show a reduced capacity to 314 bind to host cells and collagen-coated surfaces. ${ }^{38}$ Consistently, 315 


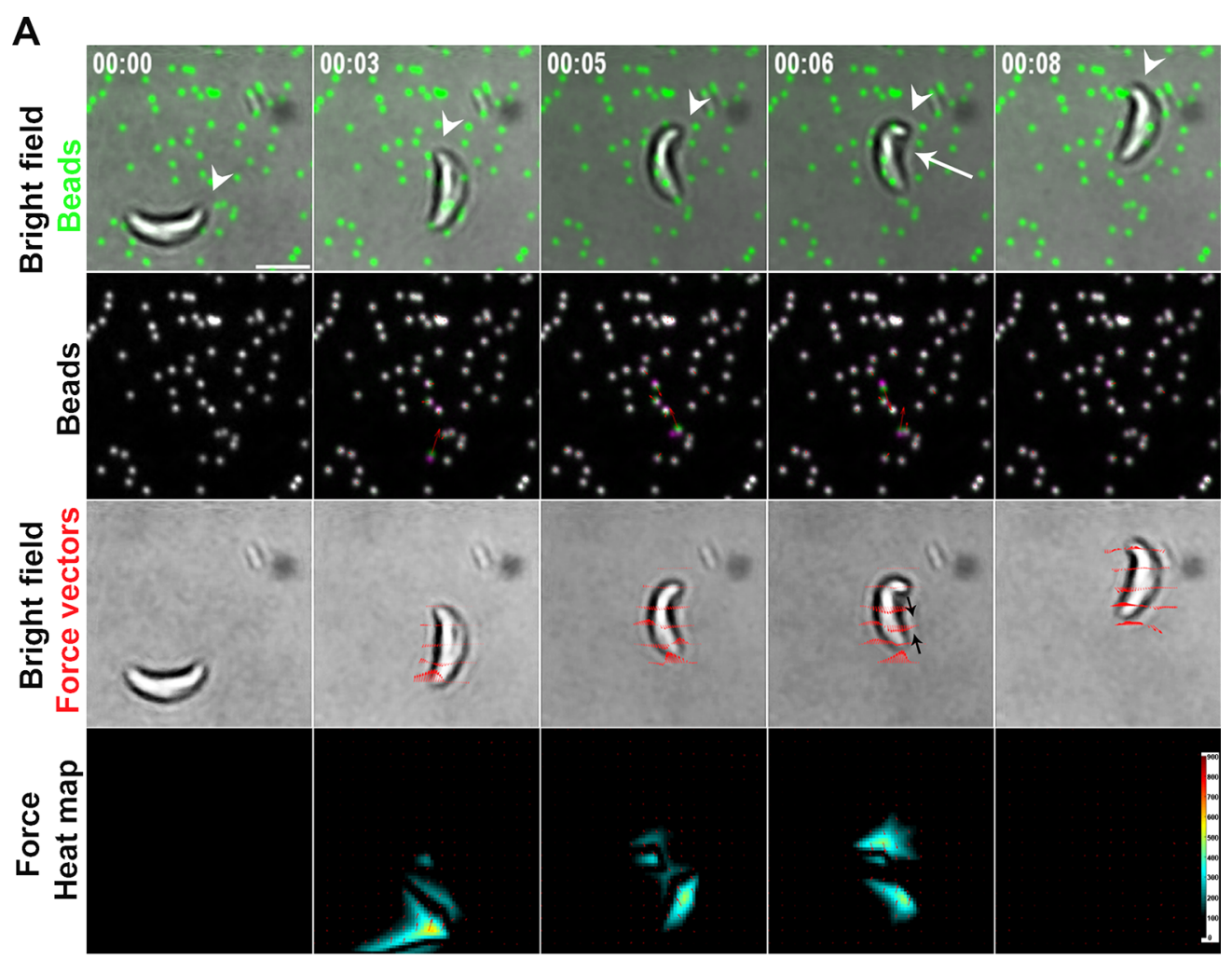

B
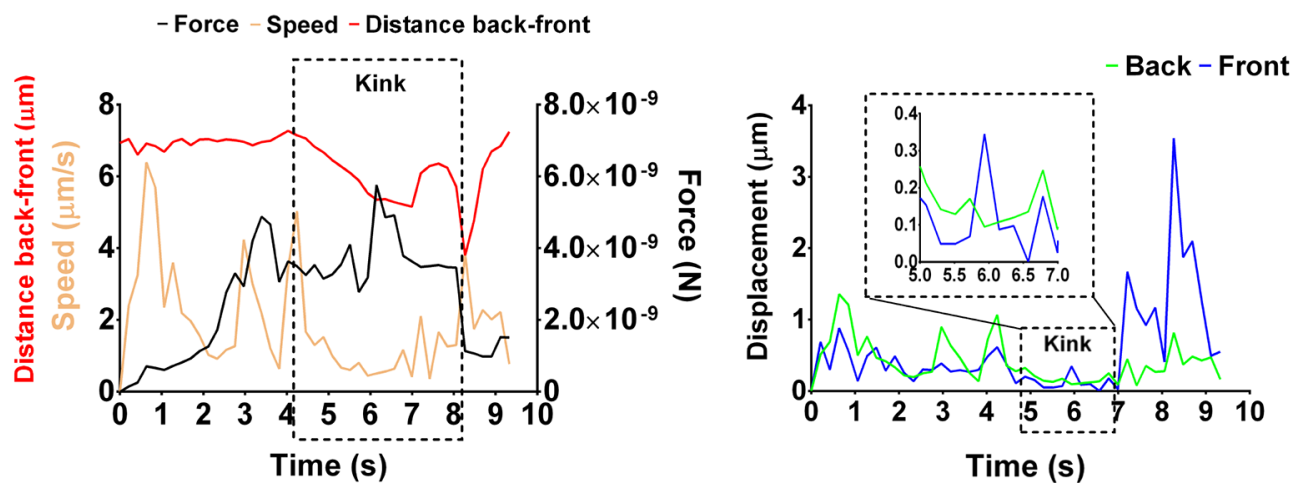

Figure 3. Helical motion includes the development of apical traction at the kink site and basal dragging forces. (A) Traction force microscopy (TFM) images were taken at the time a parasite glides on a $2 \mathrm{kPa}$ PAA gel in which fluorescent TFM beads are embedded underneath the gel surface itself coated with fibronectin $(20 \mu \mathrm{g} / \mathrm{mL})$. Panels show (top) bright-field (BF) and TFM beads composite images; white arrowheads indicate the apex of the tachyzoite, and white arrow marks the kink, (second row) beads alone, (third row) BF with force vectors computed by the MATLAB TFM code. Red arrows indicate the force direction and value; black arrows have been manually added to ease visualization of the two longitudinal forces and their orientation, (bottom) force heat map computed MATLAB TFM code. Scale bar: 5 $\mu \mathrm{m}$, time is in minutes/seconds; au for force heat map. (B) (Left) Speed (light orange line), the back to front body distance (red line) and the traction force applied by the parasite over time (black line) are shown with a zoomed inlay (black dotted line) at the kink step time, and (right) the back and front displacement (green and blue trajectories, respectively) are shown with a zoomed inlay (black dotted line) at the kink time.

316 when the $T$. gondii tachyzoite expresses only residual levels of 317 the rhomboid protease (ROM4) in charge of cleaving the 318 surface-exposed MIC2, MIC2 molecules accumulate at the 319 surface of the parasite that can no longer glide forward but 320 instead shows enhanced twirling motility. ${ }^{39}$ Similarly, when 321 Plasmodium berghei sporozoites express a mutant version of the 322 MIC2 homologue TRAP that cannot be processed by the 323 rhomboid protease, the parasite can no longer disengage its 324 posterior pole from the substrate and move forward. ${ }^{36}$ 325 Whereas we detected both SAG1 and MIC2 proteins in 326 patches over successive segments of the helical trails postfixation and immunolabeling, we did not find MIC2- 327 specific enrichment at the angular adhesion regions that were 328 accurately identified using the RICM live sequences as 329 recognition pattern (Supporting Information Figure S2). 330 Therefore, it is possible that another unknown adhesin(s), 331 which would not integrate the retrograde flow, might modulate 332 force production at the parasite apex in line with the turnover- 333 specific adhesion sites built by the Plasmodium sporozoite 334 during circular gliding. ${ }^{13}$ In addition, monitoring the apico- 335 basal flow of optically trapped beads pinned out that specific 336 adhesions do not integrate the apico-basal capping process, yet 337 
A

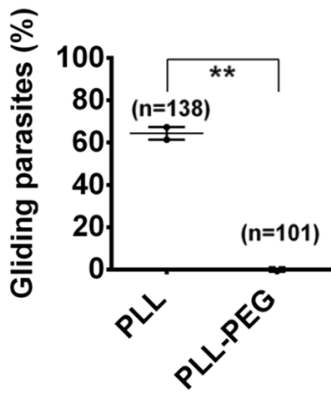

C

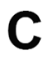

B

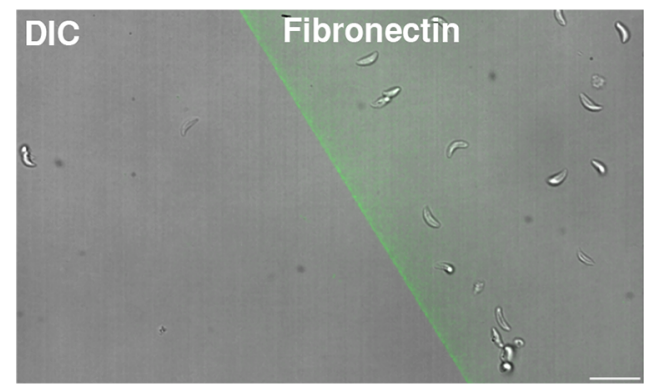

Trajectories
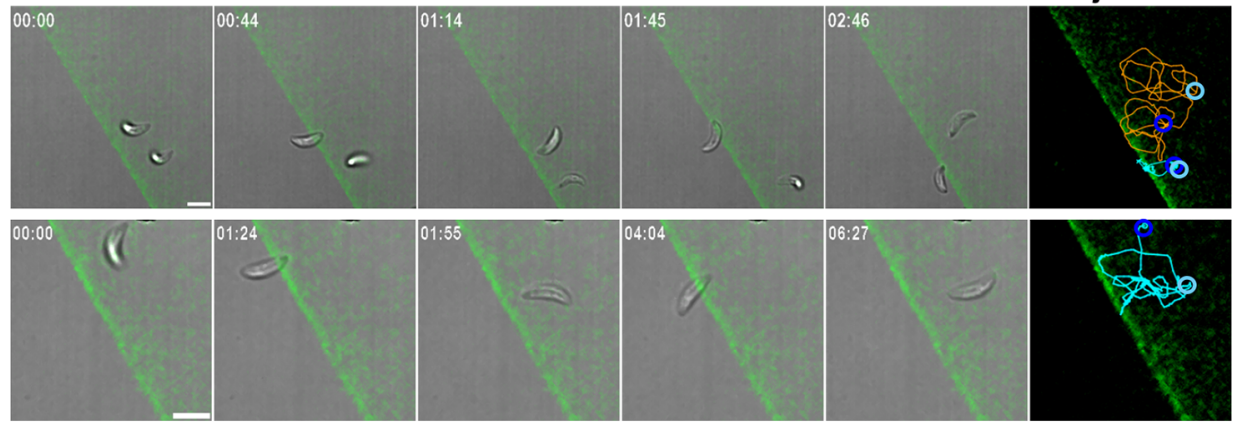

D
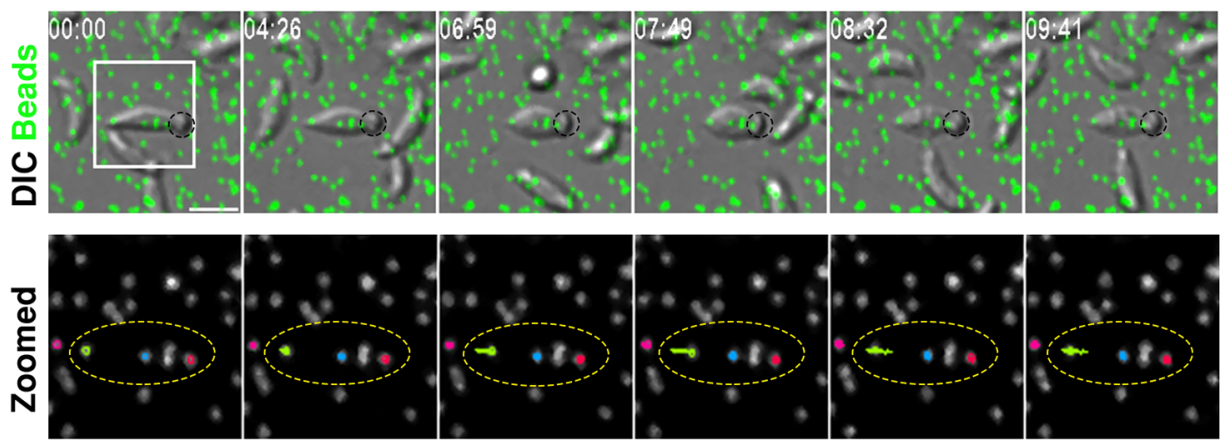

E

F
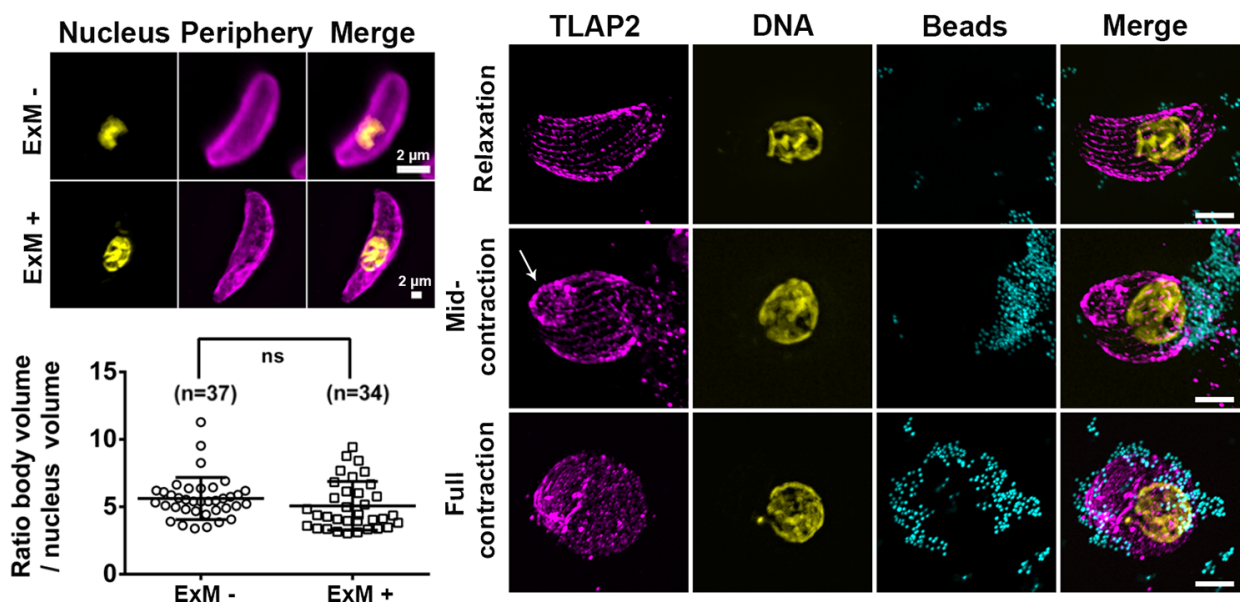

Figure 4. Front adhesion to and back disengagement from the substrate are both required for kink formation and traction force development. (A) Percentage of tachyzoites that moved by at least one body length displacement over a 20 min period post contact with poly-L-lysine (PLL) or poly(ethylene glycol) (PEG) chains grafted on PLL coating, unpaired $t$ test, $* * p=0.021$. (B) Large view of the poly-Llysine PEG-fibronectin composite micropattern shows that tachyzoites are selectively retained in the fibronectin area, which is fluorescently labeled by fibrinogen-Alexa647, scale bar: $15 \mu \mathrm{m}$. (C) Images from a sequence of two (top) or one (bottom) representative gliding tachyzoite(s) that fail to pass over the fibronectin-PLL-PEG demarcation line being incapable of interacting apically with PEG. Instead they elicit twirling around their posterior end bound to fibronectin and regain helical motion once apically sensing fibronectin. Blue and 
Figure 4. continued

cyan circles in the trajectories window indicate the initial and the final position of the tachyzoite over the several-minute gliding sequence. Trajectories were reconstituted by tracking the tachyzoite base, which unlike the apex stays in focus even during twisting and twirling motions. (D) TFM on a tachyzoite posteriorly immobilized on fibronectin through carboxylated microbeads (black circle). (Top) Selective inward displacement of TFM beads (green) beneath the apex of the immobilized parasite occurs during sustained contraction, whereas the beads relax back to initial position upon parasite relaxation, (bottom) zoomed white square from top, showing the colored trajectories; note the large yellow trajectory that attests significant bead inward displacement, the yellow dotted ellipsoid delineates the tachyzoite. (E) Confocal images showing a representative tachyzoite immunolabeled for the GAP45 protein (pink) that delineates the parasite periphery and PI stained for DNA (yellow) prior ExM (ExM-) and after ExM (ExM+), maximal projection intensity are presented, compare the scale bars that attests the physical magnification of the ExM specifmen. The graph shows the ratio of the body and nucleus volumes after $3 \mathrm{D}$ reconstruction prior (ExM-) and after ExM (ExM+), Kruskal-Wallis test, NS, $p=0,17$. (F) ExM on tachyzoites posteriorly immobilized on beads (cyan) and immunolabeled for the cortical MT-associated TLAP2 protein (pink) and PI stained for DNA (yellow), (top) no contraction has yet started, (middle) contraction has started, note the apical humpy deformation in the cMT cytoskeleton indicated with a white arrow, (bottom) cMT disruption at a latest time of tachyzoite sustained contraction. Scale bars: $5 \mu \mathrm{m}$ unless indicated, time is in minutes/seconds. The ExM allowed reaching about a $70 \mathrm{nM} x y$ resolution thanks to a $\sim 3-5$-fold increase in the biological sample size.

338 they can optimize force production. Accordingly, the trap-like 339 protein (TLP) was proposed to promote firmer adhesion to 340 the substrate by enabling specific actin remodeling underneath 341 the plasma membrane. Such function would explain why TLP 342 causes specific slowdown of the actin-dependent flow. ${ }^{31} \mathrm{With}$ 343 these in mind, we next interrogated whether the firm stable 344 apical anchor formed by the moving tachyzoite and localized at 345 the kink could indeed serve as a platform for force transmission 346 by using traction force microscopy (TFM). These assays also 347 allowed testing whether the kink-induced cell shortening could 348 be associated with tension stored in the parasite that would be 349 released to promote the thrust required for productive helical 350 movement.

351 Traction force Spatiotemporally Associates with the 352 Kink and Coincides with Body Dragging. To design the 353 TFM assay, we first identified a substrate in which the stiffness 354 was sufficient to allow helical gliding while being elastic enough 355 to measure substrate deformations via a displacement field 356 analysis performed using fluorescent beads embedded into the 357 polyacrylamide (PAA) hydrogel. Particle image velocity (PIV) 358 combined with single-particle tracking allowed analyzing 359 substrate deformation with a spatial resolution in the range 360 of tenth of nanometers. Tracking in space and time the 361 tachyzoite posterior pole and scoring as motile those that 362 performed at least one body length displacement, we found 363 that stiffer substrates such as glass $(n=73)$ and $40 \mathrm{kPa}$ PAA $(n$ $364=55)$ correlated with optimal gliding skills, whereas lowering 365 stiffness gradually decreased the frequency and duration of the 366 motile behavior. Yet, the tachyzoites retained a short-lasting 367 ability to perform helical motion on the soft $2 \mathrm{kPa}$ PAA 368 substrate $(n=103)$ (Supporting Information Figure S3). 369 Applying $\mathrm{TFM}^{40}$ to parasites gliding on $2 \mathrm{kPa}$ fibronectin370 PAA substrate, we obtained a traction vector map and 371 identified an early inward-oriented traction force stemming 372 at the cell front and exerted longitudinally in line with the 373 backward displacement of the in-gel beads (i.e., TFM beads) 374 underneath the apical part of the parasite (Figure 3A and 375 Supporting Information Movie $7 \mathrm{a}$ and Movie $7 \mathrm{~b}$, slow 376 motion). As the force progressively increased, concomitant 377 dragging of the parasite body occurred and accounted for the 378 TFM bead's displacement underneath the posterior pole in the 379 direction of the parasite motion, in line with the previous 380 detection of trails being pulled by the tachyzoite (Supporting 381 Information Movie 6). Relaxation of the TFM beads back to 382 their initial position was recorded immediately after the kink 383 release and the parasite forward acceleration (Figure 3A,B). 384 Pioneering TFM on the Plasmodium sporozoite has allowed uncovering two longitudinal forces applied at each pole during 385 circular gliding, alike what we found for the T. gondii tachyzoite 386 undergoing helical gliding. A large force localized at the 387 posterior adhesion site peaked just prior sporozoite de- 388 adhesion, whereas a lower pulling traction force at the apex 389 pointed toward the center. In contrast to what we detected for 390 the tachyzoite, large forces were also found to point 391 orthogonally toward the sporozoite center, ${ }^{13}$ where they 392 promote body stretching and thus favor close contact of the 393 whole cell with the substrate-an obligate requirement for 394 circular gliding-without contributing to force production. ${ }^{41} 395$ Therefore, although both the tachyzoite and sporozoite likely 396 use a stalling force at the back, which upon rupture of the 397 adhesive contact contributes to a thrust force, the spatiotem- 398 poral distribution and intensity of the forces during their 399 specific gliding cycle (i.e., helical versus circular) likely differ. 400 Accordingly, the overall force during the helical cycle was 401 computed to a few nanoNewtons $(\sim 1$ to $4 \mathrm{nN}, n=10)$ with an 402 increment over the kink step, therefore significantly higher 403 than the 100-200 pN range inferred for cortical forces using 404 laser trap on nongliding $T$. gondii tachyzoites ${ }^{42}$ or gliding 405 Plasmodium sporozoites. ${ }^{3}$ By comparison, the nanoNewton 406 range characterize forces applied by both bacteria and 407 mammalian cells on their surroundings. ${ }^{43}$

Toxoplasma Apical Focal Adhesion Is Essential to 409 Switch from Nonproductive Twirling to Productive 410 Helical Motion. To demonstrate the functional contribution 411 of the apical body adhesion to the subsequent helical gliding, 412 we sought interfering with the parasite adhesive step at the 413 apical traction force site. First, we screened for substrates not 414 permissive to parasite adhesion using several chemicals of 415 distinct charge and wettability properties. We found that 416 tachyzoites were unable to stabilize and glide on the densely 417 packed polymeric poly(ethylene glycol) (PEG) brushes grafted 418 on poly-L-lysine (PLL) (Figure 4A) similarly to what was $419 \mathrm{f} 4$ reported for Plasmodium sporozoites. ${ }^{44}$ Therefore, composite 420 substrate was photopatterned: the parasites underwent 421 energetic gliding on the fibronectin-coated area when 422 approaching the demarcation line between the fibronectin 423 and PLL-PEG-juxtaposed areas. The tachyzoites stopped 424 when facing PEG, sometimes sliding on it prior to stepping 425 back in a reverse motion while still posteriorly bound to 426 fibronectin (Figure 4B,C and Supporting Information Movie 8427 and Movie 9). They responded to the exclusive basal 428 attachment by spinning around the main axis in a typical 429 twirling nonproductive motion ${ }^{18}$ until the front region was 430 eventually caught again in an interaction with fibronectin, 431 
A

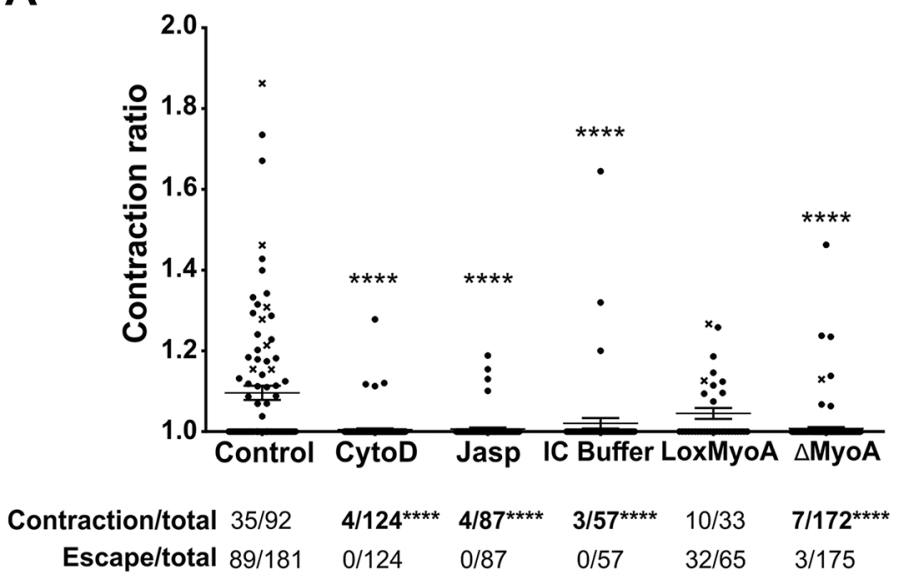

B

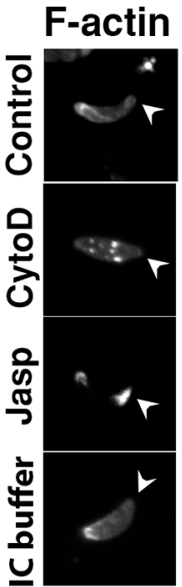

C

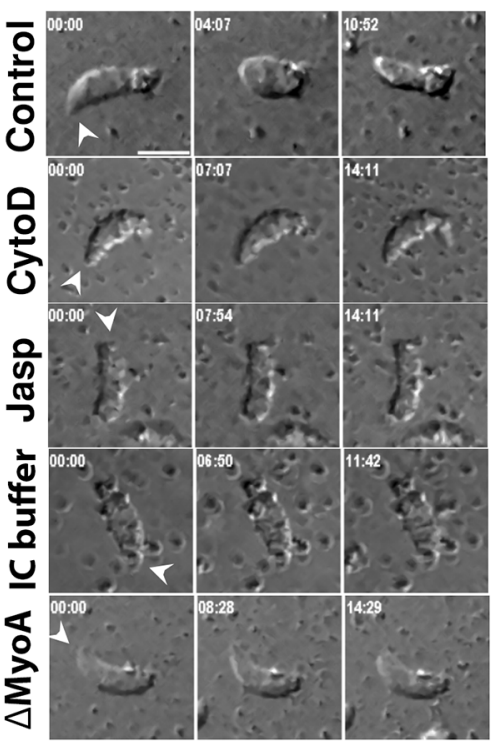

D

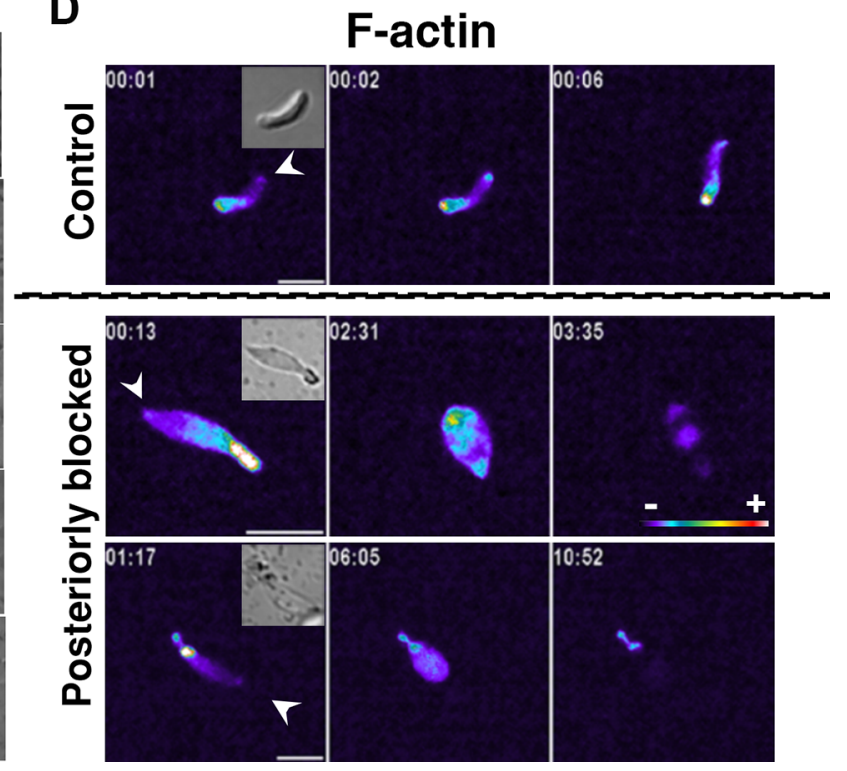

Figure 5. Prolonged and exacerbated tachyzoite contraction relies on intact actin filaments and MyoA motor. (A) Graph shows the contraction ratio after posterior immobilization on beads over a $20 \mathrm{~min}$ period for (i) wild-type control tachyzoites expressing the F-actin chromobody-Emerald (Acb-E) and incubated in EC buffer supplemented or not with $1 \mu \mathrm{M}$ cytochalasin $\mathrm{D}$ or $1 \mu \mathrm{M}$ jasplakinolide actin poisons or in IC buffer, and (ii) for tachyzoites before (LoxMyoA) and after ( $\triangle$ MyoA) Cre-mediated excision of the $m y o A$ gene. KruskalWallis test, ${ }^{* * * *} p<0.001$ Dunn's multiple comparisons with the control genotype. Table below the graph shows (i) proportions of number of parasites contracted over the total and (ii) proportions of number of parasites that escape over the total. For both, chi square test, $* * * * p$ $<0.001$ Fisher's exact test with the control. (B) Confocal images of tachyzoites showing F-actin prior to record the contractile behavior. Maximal projection intensities are presented. (C) Representative DIC images of tachyzoites posteriorly immobilized to monitor both contractile behavior and bead-capping ability under each setting over a $20 \mathrm{~min}$ period $(57>n>181$ depending on the actin poison used and $65>n>165$ for myoA KO, 3 independent assays). (D) Representative fluorescent images of F-actin dynamics in tachyzoites expressing the Ach-E undergoing helical gliding (top panel) or sustained contraction upon posterior immobilization (middle and bottom panels); DIC images are presented as inlays for each sample. Heat map intensity is presented in arbitrary units.

432 hence restoring the tachyzoite ability to perform helical 433 trajectory. These data reinforce the view that the tachyzoite 434 builds an apical stable adhesion site with the substrate that is 435 compatible with the development of traction force. As we 436 showed that both adhesion and force spatiotemporally 437 coincide with the kink-like deformation, we expected then 438 that the thrust productive force would be at least partly driven 439 by the relaxation of the kink, which drives the slight lift of the 440 parasite apex. Of note, this thrust force might also require 441 some contribution from the parasite basal contact which 442 loosens when traction operates allowing both dragging and 443 retraction of the body. Given that tachyzoites must overcome the resistance of the $3 \mathrm{D}$-nonlinear-meshwork to navigate in 444 physiological microenvironments, the acceleration induced 445 upon the kink release should provide the right thrust to pass 446 through the meshwork of fibers they pulled on. Accordingly, 447 we next addressed how the contact and dragging force at the 448 cell back identified by, respectively, RICM and TFM 449 contribute to the helical gliding mode of the tachyzoite. 450

Adhesion-De-adhesion Coupling at Both Poles 451 Drives the Toxoplasma Apical Kink and Its Release, in 452 Turn Promoting the Helical Thrust Force. We designed 453 another TFM assay in which the tachyzoite would be unable to 454 disengage its posterior pole from the substrate using submicron 455 
456 sticky beads. As already reported in the context of membrane 457 flow or cortical forces studies, ${ }^{32,42}$ the beads deposited on the 458 tachyzoite surface underwent apico-basal translocation. How459 ever, because in our setting the bead's surface engaged covalent 460 interactions not only with the parasite but also with the 461 fibronectin layer in a configuration that forced the former to 462 stay posteriorly immobilized on the latter, the cell forward 463 progression could be hindered (Figure 4D). Such posteriorly 464 constraint tachyzoites never formed the apical kink $(n=0 / 92)$ 465 and, instead, underwent a directional, minute time scale and 466 repetitive inward-oriented contraction visualized by the whole 467 body rounding (Figure 4D and Supporting Information Movie 468 10). The TFM captured the displacement of in-gel beads 469 suitably positioned underneath the apex of the immobilized 470 parasite consistent with a sustained apical traction force, and 471 therefore a sustained adhesion between the parasite front and 472 the substrate (Figure 4D, zoomed panel, yellow bead and 473 trajectory). The prolonged force application induced pro474 nounced shrinkage of the cell body along the longitudinal axis 475 and eventually led to membrane damages to the extent the 476 tachyzoite was likely wounded beyond repair. Meanwhile, we 477 observed that the in-gel beads shifted back to almost the initial 478 position, which we assumed to relate to the sudden relaxation 479 of the cell "ghost" when internal tension fell (Figure 4D and 480 Supporting Information Movie 10). Strong evidence for the 481 rupture of the plasma membrane upon excessive contraction of 482 the YFP-expressing tachyzoite was provided by the acute loss 483 of fluorescence that occurred immediately after maximal cell 484 rounding and concomitantly with the relaxation of the cell 485 (Supporting Information Figure S4). The relaxed tachyzoite 486 cell looked mis-shapen for the rest of the recording time as 487 expected for a cell ghost, therefore attesting that the 488 exacerbated and sustained contraction induced cell death. To 489 next visualize the deleterious effect of the long-lasting 490 contraction on internal cytoskeletal structures at the nanoscale 491 resolution, we applied the expansion microscopy technique $492(\mathrm{ExM})^{45}$ and first validated the isotropic expansion of the 493 specimen with the $3 \mathrm{D}$ co-reconstruction of the tachyzoite body 494 and nucleus prior and after expansion (Figure 4E). We then 495 analyzed a tachyzoite line engineered to express a fluorescent 496 version of TLAP2, a protein aligned with the $22 \mathrm{cMTs}^{30}$ and 497 confirmed that during the prolonged contraction period, the 498 capped beads accumulated posteriorly while the spiral cMTs 499 deformed. We first observed an apical hump in the cMT 500 network (Figure 4F, middle panel), and eventually, the latter 501 fell apart while the capped beads were redistributed over the 502 damaged surface (Figure 4F, bottom panel). Overall, these 503 data support that the kink rose from the interplay between the 504 apical adhesion/base de-adhesion and the forces generated at 505 both poles rather than from the apical traction force only. They 506 also highlight that creating unbalance between the front and 507 back responses can result in a contractile force that imposes a 508 mechanical compression to the cMT network to which it 509 cannot resist. The need for a balanced myosin-A-based 510 contractile activity that stems at the tachyzoite apex and 511 promotes disengagement of the posterior pole can explain why 512 the genetically myosinA-deficient tachyzoites, which retain 513 only residual motility, do not any longer undergo helical 514 gliding. ${ }^{37,32}$

515 Tachyzoite Apico-Basal Contraction Requires Intact 516 Actin Filaments and Myosin A Motor and Correlates 517 Only Partially with the Requirements for Rearward 518 Membrane Flow. Apart from the prolonged contraction and fatal outcome described earlier, we also observed that the 519 tachyzoites, which were posteriorly immobilized, could either 520 rotate on the beads, slightly contract, and move forward, hence 521 those escaped (Supporting Information Movie 11) or remain 522 inactive, hence those showed no contraction and stayed 523 attached. To verify whether actin dynamics and MyoA motor 524 the function of which are required for motility ${ }^{25,46}$ would also 525 contribute to the apically driven exacerbated contraction, we 526 first defined a quantitative readout for measuring the 527 contraction extend over time. Using the ratio between the 528 resting body length (i.e., no contraction) and the minimal body 529 length (i.e., maximum contraction) as a contraction indicator, 530 we found that nearly half of the wild-type (WT) control 531 parasites were able to escape from the immobilization setting, 532 therefore attesting to the energetically active status of the 533 specimens under study. Approximately $40 \%$ of the second half 534 $(n=35 / 92)$ contracted over a wide range of amplitude, 535 whereas $20 \%$ of this contracting population actually showed 536 signs of irreversible damages $(n=7 / 35)$ (Figure 5A). Blocking $537 \mathrm{fs}$ actin dynamics with cytochalasin $\mathrm{D}(0.5$ and $1 \mu \mathrm{M})$ or 538 jasplakinolide $(1 \mu \mathrm{M})$ that work through different mecha- 539 nisms ${ }^{47,48}$ almost abolished both the escape (i.e., motile) and 540 contractile behaviors. Of note, the few (less than 5\%) 541 tachyzoites that displayed contraction never reached the 542 stage of high and sustained body compression, hence none 543 of them died (Figure 5A). We confirmed the cytochalasin and 544 jasplakinolide potency on tachyzoite actin as parasites 545 expressing the fluorescent chromobody actin-Emerald (Acb- 546 E) ${ }^{49}$ displayed the typical drug-induced redistribution of actin 547 filaments as, respectively, cytoplasmic aggregates and apical 548 acrosome-like. ${ }^{50}$ With the same readout, we also compared the 549 contractile behavior of parasites following or not $M y o A$ gene 550 silencing using the well-characterized DiCre Lox MyoA 551 strains. $^{24}$ We observed that loss of MyoA ( $\left.\triangle M y o A\right) 552$ significantly altered the contractile capability of tachyzoites 553 immobilized on their base when compared to the MyoA- 554 expressing parental line (LoxMyoA) ( $\Delta$ MyoA $=175, p<555$ 0.001) (Figure 5A). Finally, we checked if the contractile 556 activity was specific for tachyzoites metabolically ready for 557 initiating motility by measuring the contraction of WT control 558 tachyzoites kept in the Endo buffer which mimics the 559 intracellular buffer (herafter referred to as IC buffer) and is 560 known to drastically decrease microneme secretion (i.e., 561 adhesin delivery on the tachyzoite surface) and consequently 562 motility. ${ }^{20}$ Those tachyzoites showed the typical actin posterior 563 network of intracellular tachyzoites ${ }^{51}$ and were significantly 564 inefficient at contracting under the posterior microbead setting 565 $(n \mathrm{IC}=57, p<0.001$ (Figure 5A-C and Supporting 566 Information Movie 12). Finally, we monitored actin filaments 567 in real time in Acb-E-expressing tachyzoites undergoing either 568 helical gliding or forced contraction during the immobilization 569 phase. The F-actin apical focus detected at the onset of the 570 helical cycle remained discrete and rapidly resolved into the 571 large stable basal F-actin pool, as reported ${ }^{33,51}$ and in line with 572 our force mapping assays. In contrast, an increasing F-actin 573 signal was detected within the whole body, in particular, in the 574 cell front upon prolonged contraction of the immobilized and 575 rounding tachyzoites (Figure 5D and Supporting Information 576 Movie 13). Eventually, the F-actin signal vanished, which fits 577 with the loss of fluorescence observed with YFP-expressing 578 tachyzoite artificially immobilized on the substrate through 579 their basal pole (Supporting Information Figure S4), thereby 580 confirming that the membrane had ruptured upon excessive 581 
A
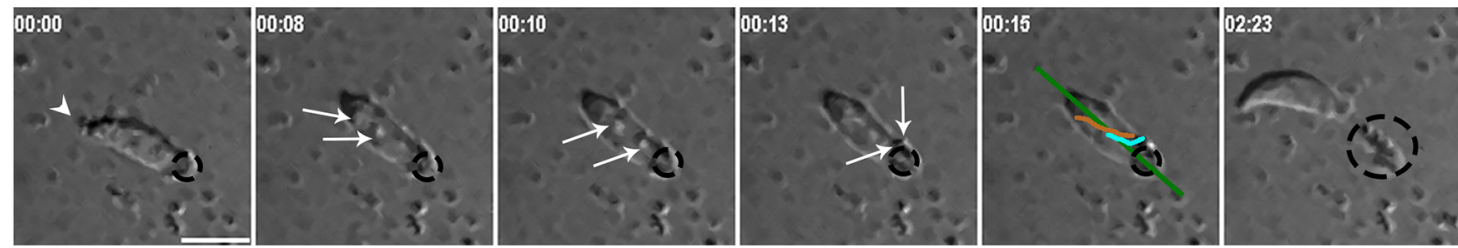

\section{B}
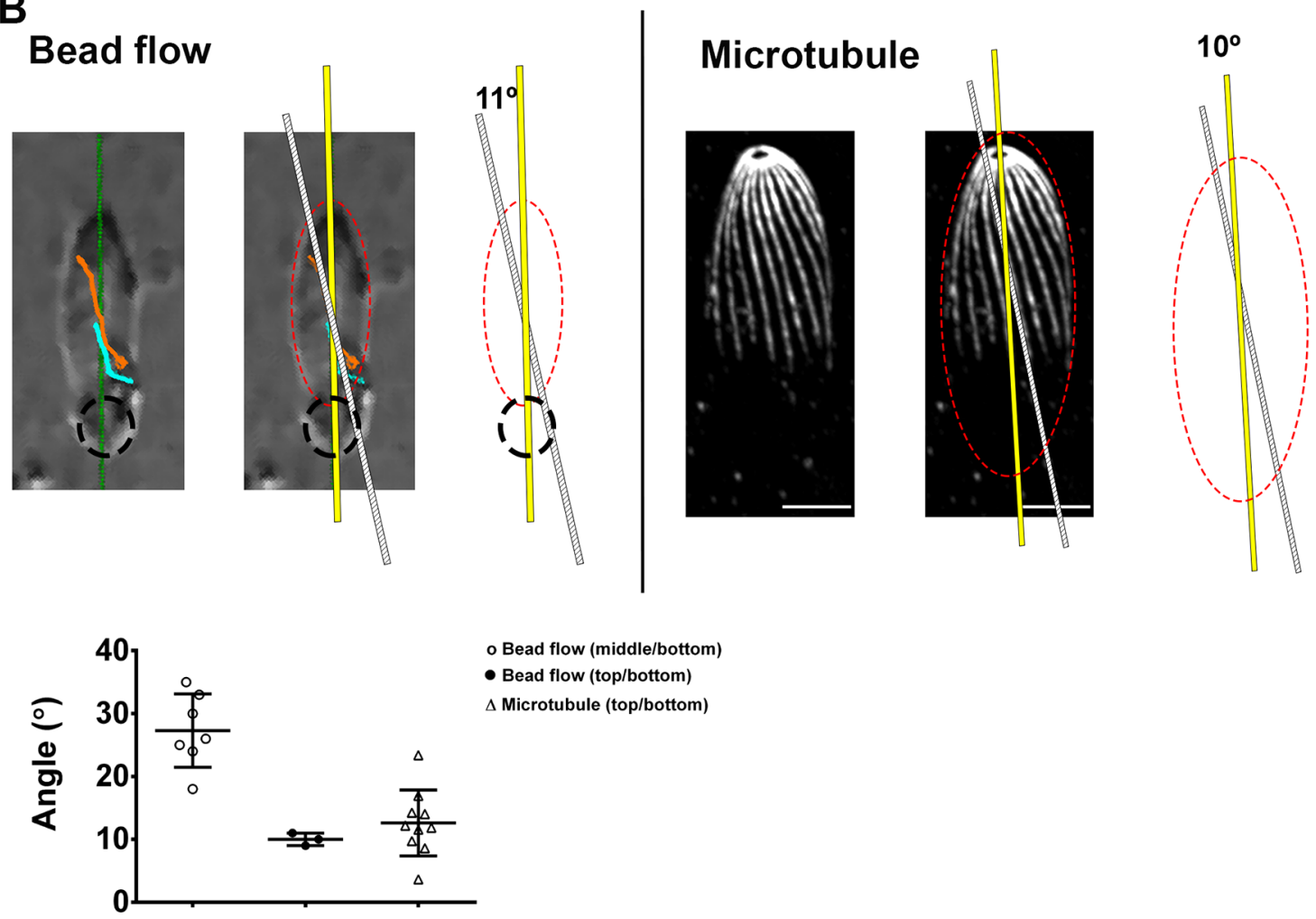

o Bead flow (middle/bottom)

- Bead flow (top/bottom)

$\Delta$ Microtubule (top/bottom)

Figure 6. Apico-basal translocation of surface-bound microbeads follows a helical path that approximates the spiral direction of the tachyzoite cMTs. (A) Representative images of apico-basal bead capping monitored in real time for tachyzoites posteriorly immobilized on beads encircled with the dotted black line. The two surface-bound beads are indicated with white arrows, and their trajectory depicted in orange and cyan lines is shown on the fifth panel, before active escape of the tachyzoite from the immobilizing setting. In all panels, white arrowheads mark the tachyzoite apex; all scale bars: $5 \mu \mathrm{m}$, time is in minutes/seconds. (B) Zoomed panels show images and schematics for (left) trajectory of two beads over the capping time taken from the sequence shown in A, which deviates from the body main axis (yellow line) of about a $11^{\circ}$ angle, while (right) the spiral cMTs visualized on an extracellular tachyzoite treated for U-ExM and stained with antipolyglutamylated tubulin show about a $10^{\circ}$ angle orientation. (Bottom) Plot of the different angles calculated for the cMTs; the mean with SD is shown for each group ( $n=10$ tachyzoites, mean of 4 cMTs per tachyzoite) and for the capping bead flow ( $n=9$ tachyzoites). Please note the larger variability shown by the bead angles, which depends on the bead position at the initial time of capture and tracking.

582 contractile activity, leaving a tachyzoite ghost, the apex of 583 which no longer attached to the substrate.

584 As the retrograde membrane flow process has been shown to 585 operate in $T$. gondii tachyzoites in absence of the actomyosin 586 system, $^{32}$ we analyzed how this process would operate for 587 tachyzoites artificially immobilized on their base. Unlike 588 previously, $^{32}$ we monitored in real time the bead-capping 589 process and found that the loss of contractility driven by actin 590 poisons and IC conditions did correlate with the inhibition of 591 the rearward bead capping along the parasite surface $(57>n>$ 592181 depending on the condition) (Figure 5C and Figure 6A 593 and Supporting Information Movie 14), thus recapitulating the 594 phenotype of formin 1(FRM1)-deficient tachyzoites ${ }^{33}$ and 595 consistent with the need of apically initiated actin filaments. 596 However, on loss of myosinA, ${ }^{46}$ whereas the tachyzoites 597 showed almost no ability to contract (Figure 5A), they intriguingly maintained a bead-capping activity almost as 598 efficient as tachyzoites from the parental line $(65>n>165) 599$ (Figure 5C and Supporting Information Movie 12), in 600 agreement with a MyoA contribution to force transmission 601 rather than production. ${ }^{32}$ Furthermore, along with these bead- 602 translocation assays we observed in WT tachyzoites that the 603 bead-capping process could precede productive helical move- 604 ment (Figure 6A and Supporting Information Movie 14), and 605 that the bead apico-basal motion could follow the cMT helical 606 trajectory. To confirm this observation, we applied ExM with a 607 recently refined near-native expansion microscopy (U-ExM) 608 protocol, $^{52}$ and labeled the $\alpha, \beta$-tubulin dimer that forms the 609 cMT polymers taking advantage of the polyglutamylation post- 610 translational modification of these isoforms. ${ }^{33}$ With this level of 611 resolution, we could measure the angle with the longitudinal 612 axis made by the cMTs when they emanate at the apical polar 613 


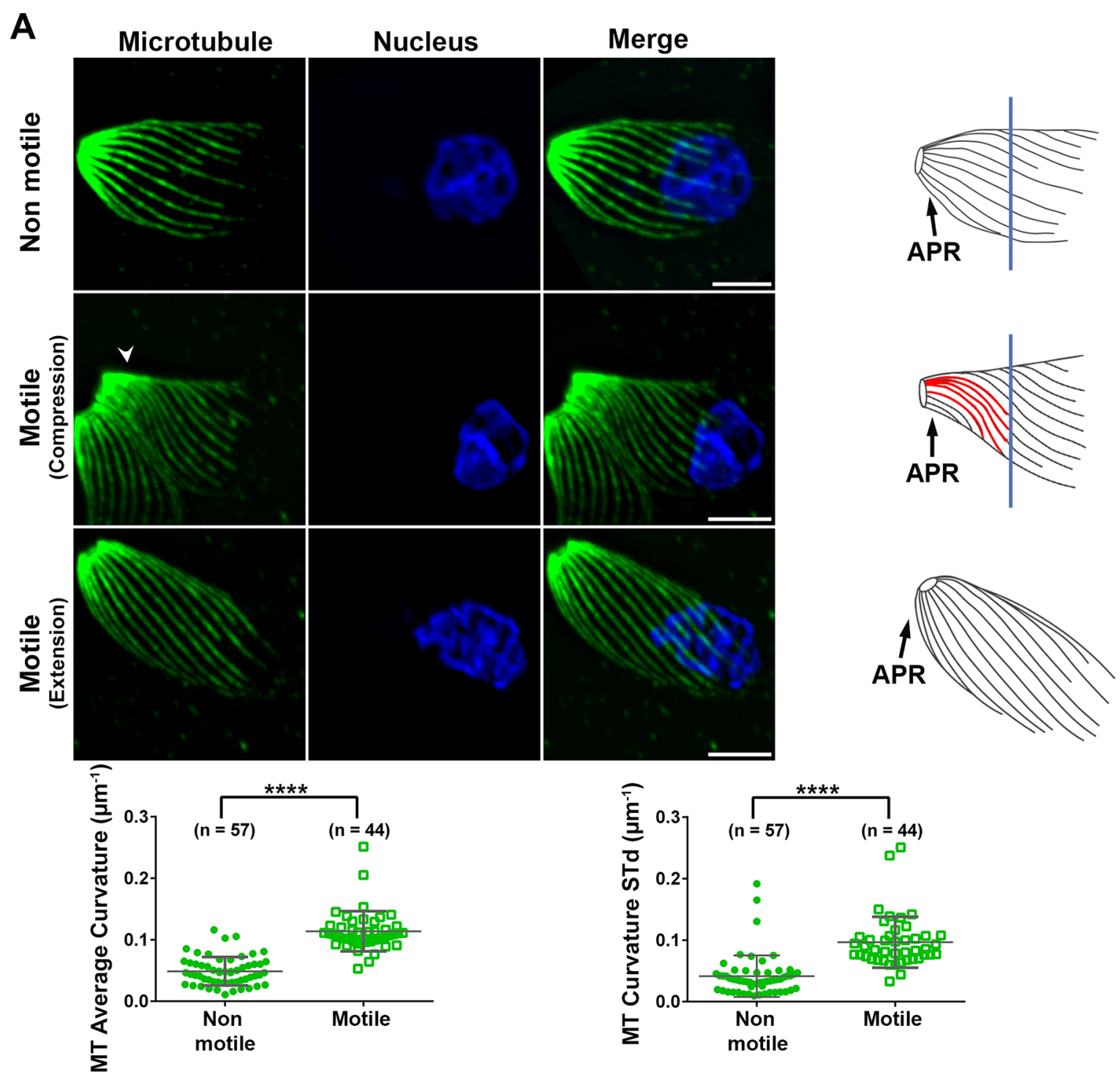

B

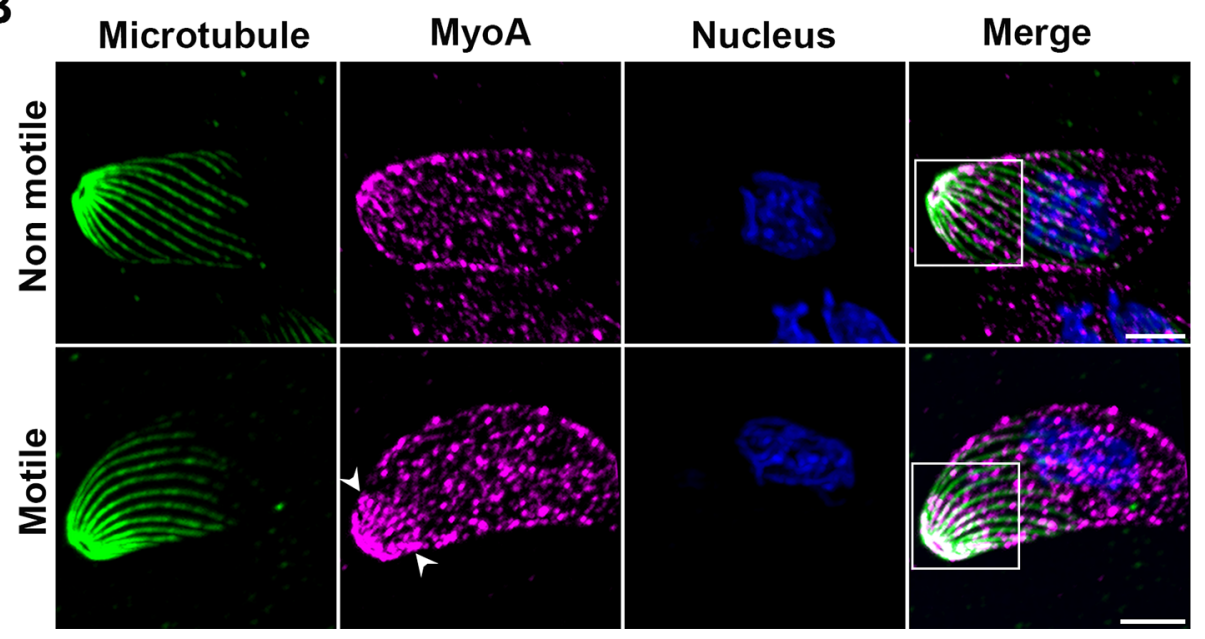

\section{Apical area}

Zoom

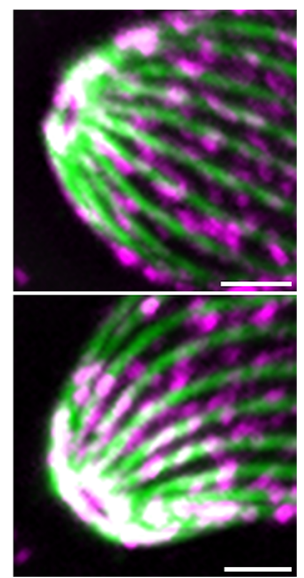

Figure 7. Curvature of cortical microtubules changes during helical gliding suggesting a spring-like mechanism apically driven by myosinbased contractile forces. (A,B) Confocal images representative of extracellular tachyzoites following U-ExM and immunofluorescence costaining of (A) tachyzoite cMTs using antipolyglutamylated tubulin antibodies (green) and nucleus (blue). Maximal projection intensities of the cMTs from the tachyzoite ventral side are shown for (top) a nonmotile tachyzoite, (middle and bottom) two motile tachyzoites. Note the pronounced torsion and compression (white arrowhead, middle panel) and the extension (white arrowhead, bottom panel) that likely account for the spring like force underlying helical gliding. Right: Schematics of the cMTs for each stage show the highly curved cMTs at the apical region of the motile tachyzoites, drawn in red. (Bottom) Graph on the left shows a significant increase in mean curvature of the cMTs 
Figure 7. continued

for the motile tachyzoites in torsion, unpaired $t$ test, $* * * p<0.0001 n=44$, when compared to the nonmotile $(n=57)$; graph on the right shows enhanced curvature amplitude over the length of single cMT for motile tachyzoites during the compression step $(n=47)$ when compared to nonmotile $(n=57)$ tachyzoites, unpaired $t$ test, $* * * p<0.0001$. (B) Co-staining of the cMTs (green) and MyoA-HA tagged using anti-HA antibodies (purple) and the nucleus (blue). (Top) Nonmotile tachyzoite, (bottom) motile tachyzoite are shown. Note the clear MyoA signal enrichment at the apex of the motile parasite indicated with white arrowheads, which co-aligns with the MT signal. The right frames show zoomed areas delineated in the merge frames with a white square. All scale bars: $5 \mu \mathrm{m}$ except for the B zoomed frames: 2 $\mu \mathrm{m}$. The U-ExM protocol gave a 3-5-fold increase of the tachyzoite size and provided a $\sim 70 \mathrm{nM} x y$ resolution. APR: Apical polar ring.

614 ring while approximating the angle made by the microbeads 615 monitored over the capping process (Figure $6 \mathrm{~B}$ and 616 Supporting Information Movie 14). Although we observed 617 for some beads that the angles were off the range of those 618 made by the well-resolved cMTs, it is worth noting that these 619 gaps mainly correspond to situations where the microbeads 620 were tracked while they had already reached the minor axis 621 plane of the ellipsoid tachyzoite. While traveling toward the 622 posterior pole, the beads approached an area devoid of cMTs 623 and often collided with the substrate before reaching the 624 posterior pole, an observation already reported when 625 monitoring the apico-basal trajectory of $0.6 \mu \mathrm{m}$ beads delivered 626 by laser trap to the surface of resting (i.e., nonmotile) 627 tachyzoites. $^{42}$ Collectively, these data indicate that the 628 actomyosin system mobilized during the rearward bead 629 translocation process has aligned with the longitudinal cMT 630 network, this independently of the apical adhesion and thus 631 before the apical traction force operates.

632 Curvature of Cortical Microtubules Significantly ${ }_{633}$ Changes during Helical Gliding, Suggesting a Spring634 like Mechanism Apically Driven by Myosin-Based 635 Contractile Forces. Triggered upon relaxation of the apical 636 kink, the extension of the tachyzoite body coincided with the 637 thrust force while the parasite rotated with an orientation 638 dictated by the MTs' helical architecture (Figure 1C and 639 Figure $3 \mathrm{~A}$ and Supporting Information Movie 3a). In 640 metazoans, the general ability of MTs to bear compressive 641 loads imposed by contractile forces translates into a 642 coordinated bending of MTs and to drive a spring-like force 643 mechanism as exemplified with the rhythmically contracting 644 cardiac myocytes. ${ }^{54}$ To interrogate whether the cMTs could 645 transiently store energy upon actomyosin activation and acts as 646 a torsional spring to promote the required thrust for helical 647 propelling, we analyzed the behavior of cMTs during helical 648 motion. Whereas the U-ExM allows nanoscale resolution, it 649 does not permit to affirm that the tachyzoites under 650 observation were actually caught in motion. As under pro651 gliding conditions, the tachyzoites displayed a statistically 652 significant elongation of the nucleus as compared to the 653 samples of either extracellular tachyzoites left in IC buffer or 654 tachyzoites which had just invaded host cells $(n>75$ for each 655 condition, Supporting Information Figure S5A), we used the 656 nucleus sphericity as first criterion. We next selected for 657 tachyzoites associated with helical trails using the surface658 exposed glycoprotein SAG1 known as robust trail marker, ${ }^{11}$ 659 which also bore an elongated nucleus $(p<0.0001, n=12$ 660 motile helical and $n=15$ intracellular) (Supporting 661 Information Figure S5B). To analyze whether individual 662 cMTs could display a specific compression, relaxation and 663 torsion behavior in motile tachyzoites, we applied U-ExM and 664 used both antiacetylated $\alpha$-tubulin and antipolyglutamylated $665 \mathrm{a}, \beta$-tubulin antibodies which have been widely used for labeling 666 T. gondii cMTs. ${ }^{53}$ When quantifying the curvature of the cMTs in nonmotile extracellular (Figure 7A, top panel) or nonmotile $667 \mathrm{f7}$ intracellular (Supporting Information Figure S5C, top panel) 668 and motile parasites (Figure 7A, middle panel; Supporting 669 Information Figure S5C, bottom panel), we observed for the 670 motile specimens, a highly significant increase in the mean 671 curvature of the MTs as compared to the nonmotile ones 672 (Figure 7A, polyglutamylated tubulin, $p<0,0001, n=57673$ nonmotile and $n=44$ motile parasites) (Supporting 674 Information Figure S5B,C, acetylated tubulin, $p<0,0001, n 675$ $=24$ nonmotile and $n=22$ motile tachyzoites). Importantly, 676 the curvature amplitude over the length of a single MT was 677 also significantly enhanced in gliding when compared to 678 intracellular parasites as assessed by the standard deviation 679 values of the curvature profile over the full length MTs (Figure 680 $7 \mathrm{~A}$, polyglutamylated tubulin, $p<0,0001$, Supporting 681 Information Figure S5B,C, acetylated tubulin, $p<0,0001) .682$ The decreased interdistance between single cMT nearby the 683 apical ring (APR) from where they emanate together with the 684 observed nonuniform bending over the cMT length strongly 685 argue for a specific compression occurring at the tachyzoite 686 apex during cell movement. In addition, situations where the 687 cMTs straightened at the apical region likely corresponded to 688 the time of the thrust force occurring upon kink release (Figure 689 $7 \mathrm{~A}$, bottom panel; see also schematics on the right). To further 690 test the hypothesis of cooperation between the actoMyoA- 691 based and the cMT-based cytoskeletons could contribute to 692 force production at the kink, we performed U-ExM imaging of 693 MyoA and cMTs simultaneously on either motile or nonmotile 694 tachyzoites. To this end, we used a tachyzoite line that 695 expresses a chimeric MyoA-HA protein under the endogenous 696 promoter and anti-HA antibodies. A clear co-alignment of the 697 MyoA and the cMTs was detected in the apical region of 698 motile tachyzoites together with a local enrichment in MyoA 699 when compared to nonmotile parasites (Figure 7B). Although 700 it is not possible to ascertain that this situation reflects the kink 701 step, the alignment between the MyoA and the cMTs makes 702 plausible an indirect connection and thus argue for an energy 703 transfer between the two cytoskeletons. In addition, this co- 704 alignment is consistent with the helical path taken by the beads 705 undergoing actin-based rearward capping. In metazoans cells, it 706 was found that the MTs were embedded in a viscoelastic 707 material provided by the surrounding medium and MT- 708 associated proteins-in particular, kinesin motors ${ }^{55}$ - that 709 influence the MT ability to buckle and bear energy load. ${ }^{54} 710$ Therefore, it would be worth investigating whether some MT 711 or/and IMC protein partners - including the APR-associated 712 kinesin, ${ }^{56}$ proteins that coat the cMT length and alveolins- 713 positioned at the apical flexure site could behave as pro-elastic 714 elements and thus optimize a cMT driven spring-like reaction. 715 Of note the intrinsic stable left-handed curvature of 716 «resting» cMTs already sign for their ability to withstand 717 large forces within the cytoplasm in agreement with their 718 contribution to shape maintenance. ${ }^{30,57}$ As the activation of 719 
720 the actomyosin system translates into force-powering motility, 721 it is plausible that the significant local increase in cMT 722 curvature of motile tachyzoites accounts for higher compres723 sive loads on the cMTs in response to the increased 724 contractility. Indeed, when the tachyzoite underwent exacer725 bated actomyosin-driven contraction (Figure 4F) in a failed 726 attempt to disengage its base from the adhesion site, the cMT 727 cytoskeleton overcame its bending capacity and eventually 728 broke up.

\section{CONCLUSIONS}

730 In this study, by combining surface sensitive biophysical 731 techniques with high resolution-high speed $2 \mathrm{D}$ and $3 \mathrm{D}$ live 732 imaging and expansion microscopy, we provided insights on 733 how the $T$. gondii parasite couples cycles of adhesion-de734 adhesion to the substrate with traction and spring-like force 735 generation to achieve helical gliding, a function which is 736 required for perpetuating $T$. gondii populations in the 737 homeothermic hosts. Owing to its persistent structural polarity 738 over the intracellular/extracellular cycles, the tachyzoite 739 bypasses the canonical symmetry break most eukaryotic cells 740 must undergo to start migrating. ${ }^{58}$ In addition, although 741 lacking the typical actomyosin II motor commonly used to 742 generate traction in eukaryotes, Toxoplasma has evolved an 743 Apicomplexa-restricted unconventional myosinA motor that, 744 immobilized within the glideosome unit and between the 745 membrane layers forming the pellicle at the cell surface, works 746 on noncanonical actin filaments to activate motility. ${ }^{29}$ The 747 pellicle and the subtending twisted 22 microtubules provide 748 the tachyzoite with a peculiar crescent shape and flexibility, 749 which has proposed to act as the primary driver of the helical 750 motion. We now demonstrate that the $T$. gondii tachyzoite 751 must engage its apical region in a specific stable adhesion with 752 the substrate that is compatible with the development of a 753 balanced traction force and drives, in turn, the loosening of the 754 posterior contact point, allowing retraction of the parasite base 755 and dragging force. Therefore, similarly to what was found for 756 the Plasmodium motile sporozoite, real time analysis of 757 adhesion dynamics and force mapping supported by recently 758 refined high spatiotemporal resolution imaging approaches and 759 biophysics characterization applied to the Toxoplasma motile 760 tachyzoite have challenged the classic model of Apicomplexa 761 gliding motility. In addition to the backward capping of 762 adhesion proteins at the cell surface, both parasite rely on a 763 distinct class of adhesive contact that do not enter the rearward 764 flow but directly contribute to build tension in the parasite 765 cytoskeleton and produce force by strengthening anchors 766 points with a specific on-off dynamics, hence regulating the 767 motile process. Although these studies confirm the need to 768 shift paradigm for a comprehensive mechanistic understanding 769 of Apicomplexa gliding motility, they raised key questions on 770 the nanoscale architecture of the singular adhesive platforms 771 including the adhesin ligand identities on the extracellular side, 772 the actin filament organization on the cytoplasmic side, but 773 also how they would transmit tensional forces from the 774 cytoskeleton to the ECM with the yet to clarify contribution of 775 the MyoA motor, and eventually, it would be worth 776 investigating their putative contribution for mechanotransduc777 tion. Providing indication of a spatiotemporally regulated 778 cooperation between the tachyzoite actomyoA-based contrac779 tile activity and the helical flexible cMT ability to build tension, 780 this study gives credit to pioneered kinematic analysis. ${ }^{18}$ These 781 results also highlight the necessity to delve deeper into the compressive, torsional, and tensile strengths of Toxoplasma 782 tachyzoite and also Plasmodium sporozoite cMTs as some 783 differences between the two stages could in part account for 784 their specific motile behavior.

785

METHODS/EXPERIMENTAL

786

Parasite Strain Maintenance and Preparation for All 787 Motility Live Assays. All media and products used for cell culture 788 were from Gibco-Life Technologies (St Aubin, France) unless 789 specified. T. gondii strains were propagated on Mycoplasma-free 790 human foreskin fibroblast monolayers (HFFs) and grown in 791 Dulbecco's modified Eagle medium (DMEM) supplemented with 792 glutamax, 10\% heat-inactivated fetal calf serum (FCS), penicillin (100 793 $\mathrm{U} / \mathrm{mL})$, and streptomycin $(100 \mu \mathrm{g} / \mathrm{mL})$ at $37{ }^{\circ} \mathrm{C}$ and $5 \% \mathrm{CO}_{2}$. The 794 laboratory type $1 \mathrm{RH}$ strain was used as well as the YFP-expressing 795 $\mathrm{RH}^{17}$ and the mutant deleted for the KU80 protein ${ }^{59}$ (gift from V. 796 Carruthers, Michigan University, USA; see Molecular Cloning 797 section). The RH $\Delta$ ku80: DiCre/lox MyoA, RH $\Delta$ ku80: DiCre/ 798 $\Delta \mathrm{MyoA}^{60}$ and the $\mathrm{RH}$ cbactinEmFP ${ }^{49}$ expressing strains were given 799 by M. Meissner (MLU Munich University, Germany) and hereafter 800 designated LoxMyoA, $\triangle \mathrm{MyoA}$, or Ach-E, respectively. For most 801 motility assays, tachyzoites were collected within a few hours 802 following spontaneous egress from the HFF monolayers, and $\sim 10^{5} 803$ cells were centrifuged in $\mathrm{HBSS}^{++}$supplemented with $0.2 \%$ FCS $(0.2 \% 804$ HBSS). The pellet containing parasites was resuspended in $150 \mu \mathrm{L}$ of 805 $1 \%$ FCS HBSS (1\% HBSS) and adjusted at $1.6 \mathrm{mM}$ of $\mathrm{CaCl}_{2}(1 \% 806$ HBSS-Ca ${ }^{2+}$ ). Typically, when using the $18 \mathrm{~mm}$ diameter glass 807 coverslip fitting chamber, $50 \mu \mathrm{L}$ of the tachyzoite suspension was 808 added to $100 \mu \mathrm{L}$ of $1 \%$ FCS HBSS-Ca ${ }^{2+}$ covering the coverslip. The 809 amounts of $\mathrm{FCS}, \mathrm{CaCl}_{2}$, and pharmacological reagents were adjusted 810 upon need as described in each appropriate section.

Molecular Cloning. A mCherry (mC) tag was fused in frame with 812 the C-terminus of TLAP2 gene (ToxoDb.org, TGGT1_232130) in 813 the $\Delta \mathrm{Ku} 80$ strain using the ligation independent cloning strategy. ${ }^{59} \mathrm{~A} 814$ $1.5 \mathrm{~kb}$ fragment corresponding to the $3^{\prime}$ end of the TLAP2 excluding 815 the stop codon was amplified by PCR with the forward primer $5^{\prime}-816$ GCGCCCTCCTTCAGTGTTCTTC-3' and reverse primer 5'- 817 TGCACCCTGCGGCACCGGCAG-3'. The PCR fragment and 818 vector p-LIC-mCherry-HXG were digested with T4 DNA polymerase 819 and annealed, yielding plasmid pTLAP2mCherry-LIC-DHFR. Freshly 820 egressed $\Delta$ ku80 tachyzoites from a $1 / 2$ T25 flask were transfected 821 with $15 \mu \mathrm{g}$ of the EcoRV-linearized plasmid. After one cycle without 822 drug, $\triangle \mathrm{Ku} 80 / \mathrm{TLAP} 2-\mathrm{mC}$ transformants were selected with $2 \mu \mathrm{g} / \mathrm{mL} 823$ pyrimethamine and single-cell cloned by limiting dilution. 824

Endogenous tagging of MyoA with HA-tag was achieved using the 825 pLIC-MyoA-HA-HXGPRT vector. To construct this plasmid the 826 coding sequence of MyoA was amplified using primers 5'-827 TACTTCCAATCCAATTTAGCAAAGCAGCAAACTGCCCA- 828 GCGAAG-3' and 5'-TCCTCCACTTCCAATTTTAGC- 829 GAACGCCGGCTGAACAGTCGCGGG-3' and RH $\Delta k u 80$ genomic 830 DNA as template. The PCR product was cloned to pLIC-mAID-HA- 831 HXGPRT vector using the LIC cloning method as described above. ${ }^{61} 832$ RHku80 OsTir1-(Ty)3 parasites were electroporated with BstBI- 833 linearized plasmid, and recombinant parasites were selected with 834 mycophenolic acid $(25 \mu \mathrm{g} / \mathrm{mL})$ and xanthine $(50 \mu \mathrm{g} / \mathrm{mL})$. Stable 835 transgenic parasites were single-cell cloned by limiting dilution. $\quad 836$

Video Microscopy. Time-lapse video microscopy was conducted 837 in Chamlide chambers (LCI Corp., Seoul, Korea) accommodating 18838 $\mathrm{mm}$ diameter coverslips or $35 \mathrm{~mm}$ MatTek glass bottom dishes 839 (Matek corporation) installed on an Eclipse Ti inverted confocal 840 microscope (Nikon France Instruments, Champigny sur Marne, 841 France) set up with a temperature and $\mathrm{CO}_{2}$-controlled stage and 842 chamber (LCI Corp., Seoul, Korea). The microscope was equipped 843 with the sCMOS prime camera (Photometrics), a $60 \times$ objective, and 844 a CSU X1 spinning disk (Yokogawa, Roper Scientific, Lisses, France). 845 MetaMorph software was used for controlling the microscope 846 (Universal Imaging Corporation, Roper Scientific, Lisses, France). 847 All live microscopy was performed at $37{ }^{\circ} \mathrm{C}$ and $5 \% \mathrm{CO}_{2}$. 
849 Image Processing. For image processing, we combined the use of 850 ImageJ, Icy, MATLAB, ChimeraX, and Amira-Avizo (Thermo-Fisher) 851 software. Details of image processing are included in the appropriate 852 protocol sections. Slow motion videos were created using Adobe 853 Premiere with the optical flow module.

854 Motility Assays in Collagen 1 Fibrous Matrices. Collagen gels 855 were made using rat tail type I collagen (CellSystems). Nine parts of 856 collagen dissolved in acidic medium were mixed with 1 part of 857 neutralization solution to reach the concentration of $4 \mathrm{mg} / \mathrm{mL}$ and 858 promote matrix formation. All solutions were kept in $4{ }^{\circ} \mathrm{C}$ during 859 preparation. Then dilution of the gel at a concentration of $2 \mathrm{mg} / \mathrm{mL}$ 860 in $\mathrm{HBSS}^{++}$supplemented with $1 \%$ FCS was rapidly spread in the 861 middle of a MatTek glass bottom dish and incubated for $30 \mathrm{~min}$ at 37 $862{ }^{\circ} \mathrm{C}$ and $5 \% \mathrm{CO}_{2}$. Labeling of the collagen fibers was achieved with the 863 recombinant CNA35-EGFP produced in Escherichia coli (BL21-DE3 864 strain) used at $2 \mu \mathrm{M}$ final concentration according to the protocol. ${ }^{35}$ 865 A $50 \mu \mathrm{L}$ volume of the tachyzoite suspension prepared in $1 \%$ FCS $866 \mathrm{HBSS}^{-\mathrm{Ca}^{2+}}$ was deposited on top of the gel prior to being transferred 867 in the microscopy stage for video microscopy. Once tachyzoites had 868 penetrated within the mesh, images were captured at 1 frame/s. For 869 tracking the fibers during parasite navigation, we used Icy and 870 visualized with green the initial position of the fibers at $t=0 \mathrm{~s}$ while 871 we duplicated this frame as many times as the number of the time872 lapse frames. Then we merged this "time zero" stack with the original 873 time lapse in which the fibers were colored in magenta. The whitish 874 color accounts for the green and magenta overlay and indicates no 875 displacement, whereas the detection of the green and magenta reveals 876 the fiber displacement during the sequence.

877 Tachyzoite 2D Gliding Assays. Parasites were prepared as 878 described above, and assays were carried on $18 \mathrm{~mm}$ plasma-activated 879 glass coverslips coated with either fibronectin $(20-50 \mu \mathrm{g} / \mathrm{mL}$ in 880 phosphate-buffered saline (PBS)) or poly-L-lysine $(150-300 \mathrm{kDa}$ $881 \mathrm{PLL}, 50 \mu \mathrm{g} / \mathrm{mL}$ in PBS). Image analysis included tracking the 882 tachyzoite back and front parts over the gliding time by using Icy as 883 described. ${ }^{62}$ "Manual tracking" and "Motion profiler" plugins were 884 used to extract the $x-y$ position of the back and front spots over time. 885 Having tracked the $x-y$ positions over time, we used the "Distance 886 profiler" plugin to calculate the distance between the two positions 887 (back and front) over time.

888 Reflection Interference Contrast Microscopy. RICM images 889 were acquired on an inverted microscope (IX71, Olympus) on which 890 a white-light source (HPLS 345, Thorlabs) was coupled using a 891 home-built illumination arm incorporating a diaphragm (SM1D12C, 892 Thorlabs) controlling the illumination numerical aperture $(\mathrm{NA}=0.46$ 893 in all experiments shown here) and a triple-band spectral filter (FF01$894457 / 530 / 628-25$, Semrock) as described. ${ }^{63}$ The usual fluorescence 895 cube in the microscope turret was replaced by polarization optics, 896 allowing separation of the incoming and reflected light (polarizer 897 WP25M-VIS, Thorlabs), polarizing beamsplitter cube (PBS251, 898 Thorlabs), and achromatic imaging flat quarter waveplate (QWP; 899 custom-made, Fichou, France). The sample was imaged with an oil900 immersion objective (60XO UPLSApo, Olympus, Japan) onto a 901 sCMOS camera (Orca Flash 4 v2, Hamamatsu) after passing through 902 an autofocus device (CRISP system, ASI imaging, USA) and a home903 built image-splitting setup incorporating a variable slit (VA100/M, 904 Thorlabs), achromatic relay lenses (ITL200, Thorlabs), imaging flat 905 dichroic mirrors (FF484-FDi01-25X36 and FF560-FDi01-25X36, 906 Semrock) gently held through curable silicon paste (Sugru, Form907 FormForm, UK), silver mirrors (PFSQ10-03-P01, Thorlabs) and 908 dichroic filters (blue: Semrock FF01-452/45-25; green: FF01-531/46909 25; red: FF01-629/56-25, Semrock). In addition, for bright-field (BF) 910 imaging, a red colored glass filter (FGL610S, Thorlabs) was used to 911 filter the light for the microscope illumination arm that was 912 subsequently focused by a long-distance 0.3 NA condenser (all 913 Olympus, Japan). BF and RICM images were acquired simulta914 neously, in the red channel, and thus show a dim RICM image 915 superimposed. Finally, a custom-built thermostated box (Digital Pixel, 916 UK) enclosing most of the microscope was used to control the 917 temperature at the sample. Image processing was done using ImageJ. 918 Blue, green, and red stacks of images were first manually registered.
RICM images were subsequently filtered using the "FFT band-pass 919 filter" plugin (1-100 pixels) to remove high-frequency noise and 920 uneven illumination background. The two (in the case of 921 simultaneous BF/RICM imaging) or three (in the case of only 922 RICM imaging) RICM images were then superimposed and 923 converted into a RGB image. BF images and RGB RICM images 924 were then segmented using the "trainable Weka segmentation" 925 plugin. ${ }^{64}$ For $\mathrm{BF}$ images, the classifier was trained to segment the 926 whole parasite, and the outline of the resulting binary image was 927 extracted subsequently. For RICM images, the algorithm was used to 928 separate the "close contact" area defined by the first dark RICM fringe 929 on the parasite surface, the trail (when relevant), the rest of the RICM 930 signal coming from the parasite (when no BF image was present), and 931 the rest of the image. Because the classifier could detect the second 932 dark RICM fringe manual correction of the segmented images was 933 performed afterward to remove incorrect areas classified as "close 934 contact". A binary image was obtained for the "close contact" area, 935 whereas an 8-bit probability image was extracted for the trail. Analysis 936 of the processed images was also performed using ImageJ. The 937 kymograph was obtained using the plugin "KymoResliceWide". For 938 measuring the speed of the parasite back end, the corresponding white 939 line on the kymograph was isolated and the positions of the pixels 940 were exported to Origin. After smoothing, the position curve was 941 derived to obtain the velocity of the back end over time. 942

Traction Force Microscopy. TFM setting was adapted from ref 943 65. The $2 \mathrm{kPa}$ PAA gels were made and mixed with TFM beads 944 (FluoSpheres carboxylate-modified microspheres, ThermoFisher 945 Scientific, $0.2 \mu \mathrm{m}$ dark red fluorescent, $\lambda: 660 / 680,2 \%$ solid). The 946 gel was top-coated with fibronectin $\left(20 \mu \mathrm{g} / \mathrm{mL}, 30 \mathrm{~min}, 23{ }^{\circ} \mathrm{C}\right) 947$ diluted in sodium bicarbonate $\mathrm{pH} 8.3(100 \mathrm{mM})$. Typical samples 948 were made from stocks of $0.7 \mu \mathrm{L}$ beads mixed in $165 \mu \mathrm{L}$ of gel 949 supplemented with $1 \mu \mathrm{L}$ of both tetramethylethylenediamine 950 (TEMED) and ammonium persulfate (APS) $10 \%$ using $20 \mu \mathrm{L}$ of 951 bead-gel suspension for an $18 \mathrm{~mm}$ diameter silanized glass coverslip. 952 A $50 \mu \mathrm{L}$ volume of the tachyzoite suspension prepared in $1 \%$ HBSS- 953 $\mathrm{Ca}^{2+}$ (see above) was deposited on top of the fibronectin-coated PAA 954 gel already filled with $100 \mu \mathrm{L}$ of $1 \% \mathrm{HBSS}-\mathrm{Ca}^{2+}$ in the appropriate 955 chamber and video recording started upon tachyzoite contact with the 956 substrate. MetaMorph streaming option was used for fast recording 957 $(<1 \mathrm{frame} / \mathrm{s})$ in both the DIC and far-red channels. Analysis of the 958 TFM beads displacement for extracting the force values carried out 959 using MATLAB. The TFM bead displacement was assessed using as a 960 reference bead position, the frame with a gel area free of parasite (i.e., 961 relaxed position of the gel). Then using the frames taken over time, 962 the bead's displacement was tracked from their initial relaxed position. 963 To check the tachyzoite gliding behavior on a substrate of different 964 stiffness, gels of different PAA concentration and coated with a layer 965 of fibronectin were made using the same protocol, omitting the bead 966 addition step, and the stiffness was controlled by atomic force 967 microscopy.

968

Micropatterned Devices. Micropatterned PEG-fibronectin 969 composite coverslips were engineered by Alveole using the PRIMO 970 patterning with specific area coated with a mix of fibronectin $(100 \mu \mathrm{g} / 971$ $\mathrm{mL})$ and AlexaFluor647-coupled fibrinogen $(10 \mu \mathrm{g} / \mathrm{mL})$ and other 972 coated with PLL-PEG. Fifty microliters of the tachyzoite suspension 973 prepared in $1 \%$ HBSS-Ca ${ }^{2+}$ was deposited on top of the fibronectin- 974 coated glass coverslip placed in the chamber and prefilled with $100 \mu \mathrm{L} 975$ of buffer prior to video microscopy.

Rear-Blocked Parasite Assay. The $0.2 \mu \mathrm{m}$ diameter beads 977 (FluoSpheres carboxylate-modified microspheres, ThermoFisher 978 Scientific F8807, $\lambda$ : 660/680, 2\% solid) were activated using the 979 microsphere coupling-two-step EDC/Sulfo NHS covalent coupling 980 procedure for Estapor carboxyl-modified dyed microspheres protocol 981 (MerckMillipore). Fifty microliters of the stock-activated bead 982 solution was diluted in $150 \mu \mathrm{L}$ of MES buffer ( $50 \mathrm{mM}, \mathrm{pH} 6.0$ ). 983 Then $30 \mu \mathrm{L}$ of the bead solution was added to $120 \mu \mathrm{L}$ of the 984 tachyzoite suspension in $0.15 \%$ FCS $\mathrm{HBSS}^{++}$(0.15\% HBSS). Fifty 985 microliters of the parasite suspension (from either the RH $\Delta \mathrm{Ku} 80,986$ $\Delta \mathrm{MyoA}, \mathrm{RH} \Delta \mathrm{Ku} 80 \mathrm{Acb}-\mathrm{E}$, or YFP tachyzoite lines) was deposited on 987 the $(50 \mu \mathrm{g} / \mathrm{mL})$ fibronectin-coated glass coverslips in the microscopy 988 
989 chamber, which was already filled with $200 \mu \mathrm{L}$ of $0.15 \% \mathrm{HBSS}^{++}$. In 990 some experiments, parasites were preincubated for $10 \mathrm{~min}$ with 0.5 or $9911 \mu \mathrm{M}$ of either jasplakinolide or cytochalasin D (Sigma) and video992 recorded in the presence of the compounds. For the intracellular 993 mimicking conditions, tachyzoites collected by centrifugation in $9940.15 \% \mathrm{HBSS}^{++}$were rinsed in ENDO buffer referred to here as IC 995 buffer (145 mM KCl; $5 \mathrm{mM} \mathrm{NaCl} ; 1 \mathrm{mM} \mathrm{MgCl}_{2}$; $15 \mathrm{mM} \mathrm{MES;} 15$ 996 mM HEPES; pH 8.3 $)^{66}$ before use, and the activated beads were 997 prepared in IC buffer. The mixture prepared as mentioned above was 998 deposited on the microscopy chamber, which was prefilled with 200 $999 \mu \mathrm{L}$ of IC buffer. Images were acquired at $1 \mathrm{~s}$ intervals for $20 \mathrm{~min}$. To 1000 describe the contractile potential of tachyzoites under each 1001 experimental condition, we calculated the contraction ratio by 1002 measuring two times the length of the parasite body starting from 1003 the back until its farther apical point but excluding the conoid to avoid 1004 false measurements in case of fluctuating extrusion. The first measure 1005 corresponded to the relax position and the second to the maximal 1006 contraction. Division of the relaxed versus the contracted length 1007 provided the contraction ratio. When needed, both DIC and 1008 fluorescence channels were recorded at $\sim 1$ frame/s.

1009 Bead Flow Assays. The assay was performed similarly to the rear 1010 blocked parasite assay. For tracking the beads, visualizing their tracks 1011 and extracting their speed, "Spot detector" and "Track manager" 1012 plugins (Icy) were used.

1013 Expansion Microscopy. To analyze gliding tachyzoites, we 1014 collected the parasites in either in prewarmed 1\% FCS HBSS-Ca ${ }^{2+}$ 1015 or IC buffer, which were centrifuged at low speed to synchronize their 1016 sedimentation and left to glide for $10 \mathrm{~min}$ on PLL $(50 \mu \mathrm{g} / \mathrm{mL})$-coated 1017 plasma-activated glass coverslips $\left(37{ }^{\circ} \mathrm{C}, 5 \% \mathrm{CO}_{2}\right)$. Gliding was 1018 stopped by addition of paraformaldehyde (PFA, 3.2\%, $15 \mathrm{~min}$ ). The 1019 tachyzoite surface and trails were immunolabeled with anti-TgPSAG1 1020 antibodies (mAb, clone TP3), and the chimeric TLAP2 protein was 1021 detected using the rabbit anti-mCherry antibodies (Institut Curie, 1022 TAb-IP Paris, France) directed against the $\mathrm{mC}$ tag after cell 1023 permeabilization with TritonX-100 ( $0.1 \%$ in PBS, $5 \mathrm{~min})$. The 1024 samples were next incubated with appropriate secondary highly cross1025 adsorbed antibodies $(4 \mu \mathrm{g} / \mathrm{mL}, 2 \mathrm{~h})$. The ExM protocol was adapted 1026 from ref 45 . Briefly Acryloyl-X was used at $0.1 \mathrm{mg} / \mathrm{mL}$ PBS for $2 \mathrm{~h}$ at $102723{ }^{\circ} \mathrm{C}$ prior to the gelation step, which was performed by placing the 1028 gelation solution $(80 \mu \mathrm{L})$ on parafilm and the coverslip with the cell 1029 sample face down on top of it. Gelation was allowed to proceed at 37 $1030{ }^{\circ} \mathrm{C}$ for $1 \mathrm{~h}$ followed by the digestion step (0.5\% Triton X-100, 8 units/ $1031 \mathrm{mLProteinase} \mathrm{K}$ ) carried out overnight at $23^{\circ} \mathrm{C}$. A $4 \mathrm{~mm}$ diameter gel 1032 core sample was collected with a puncher and immerged in $5 \mathrm{~mL}$ of $1033 \mathrm{ddH}_{2} \mathrm{O}$ in a $50 \mathrm{~mm}$ diameter Petri dish to promote expansion, while 1034 PI staining was concomitantly performed (2.5 $\mu \mathrm{M}$ in PBS). $\mathrm{H}_{2} \mathrm{O}$ was 1035 exchanged every $20 \mathrm{~min}$ until maximal expansion. The piece of gel 1036 was next gently transferred onto a PLL-coated glass coverslip in the 1037 minimal liquid volume to avoid gel drift. The $0.2 \mu \mathrm{m} Z$-image stacks 1038 of parasites in stages were captured using a spinning disk confocal 1039 Eclipse-ti Nikon microscope.

1040 Ultrastructure Expansion Microscopy. Gliding assays were 1041 performed with $\mathrm{RH} \Delta \mathrm{Ku} 80 \mathrm{MyoA}-\mathrm{HA}$ similarly to that for ExM. 1042 Additionally, for the invasion assay, HFF cells were plated on a poly-L1043 lysine-coated glass coverslip to obtain $80 \%$ cell confluence on the 1044 following day. Parasites were settled on top of the cells by gentle 1045 centrifugation $(2 \mathrm{~min}, 250 \mathrm{~g})$ and left to invade for $1 \mathrm{~h}\left(37^{\circ} \mathrm{C}, 5 \%\right.$ $1046 \mathrm{CO}_{2}$ ). For U-ExM, we followed the protocol developed by the 1047 Guichard laboratory, ${ }^{52}$ but to optimally preserve both free and 1048 intracellular tachyzoite shapes, we found it better to fix the samples 1049 before expansion in a solution made of $0.7 \%$ formaldehyde and $1 \%$ 1050 acrylamide in PBS $\left(4-5 \mathrm{~h}, 37^{\circ} \mathrm{C}\right)$. Next, similar to the ExM protocol, 1051 gelation was carried out for $1 \mathrm{~h}\left(37^{\circ} \mathrm{C}\right)$. Coverslips with gels were 1052 then transferred into $\sim 2 \mathrm{~mL}$ of the denaturation buffer in a 6-well 1053 plate for $15 \mathrm{~min}\left(23^{\circ} \mathrm{C}\right)$. Gels were removed from the coverslips with 1054 tweezers and transferred to a $35 \mathrm{~mm}$ diameter glass Petri dish filled 1055 fresh denaturation buffer for a $60 \mathrm{~min}$ incubation at $95{ }^{\circ} \mathrm{C}$. After 1056 denaturation, gels were placed in a $150 \mathrm{~mm}$ diameter glass Petri dish 1057 filled with $\mathrm{ddH}_{2} \mathrm{O}$ for the first expansion. Water was exchanged at 1058 least twice every $30 \mathrm{~min}$ at room temperature, and gels were incubated overnight in $\mathrm{ddH}_{2} \mathrm{O}$. After limited shrinkage in PBS, several 1059 $4 \mathrm{~mm}$ diameter pieces of gel were collected and deposited on 24-well 1060 plates. Gels were then incubated for $3 \mathrm{~h}$ at $37^{\circ} \mathrm{C}$ under shaking in 1061 primary antibodies (antiacetylated $\alpha$-tubulin, mAb, clone 6-11B-1) or 1062 rabbit $\alpha$-polyglutamylated (mAb, clone GT335) and rat anti-HA 1063 antibodies (mAb, clone 3F10). Gels were washed in PBS-Tween $0.1 \% 1064$ prior to incubation with appropriate secondary highly cross-adsorbed 1065 antibodies $(4 \mu \mathrm{g} / \mathrm{mL})$ for $150 \mathrm{~min}$ at $37^{\circ} \mathrm{C}$ and washed again in PBS- 1066 Tween $0.1 \%$. Expansion was achieved by controlled immersion in 1067 $\mathrm{ddH}_{2} \mathrm{O}$. The PI staining, final $\mathrm{ddH}_{2} \mathrm{O}$ wash, and gel mounting steps 1068 were all performed as mentioned for ExM.

ExM and U-ExM Image Processing. 3D reconstruction was 1070 achieved with the UCSF ChimeraX software from the raw data (i.e., 1071 xyz files) processed with ImageJ to first crop the region of interest and 1072 then to apply the "Iterative Deconvolve 3D" plugin for each channel. 1073 We used the ImageJ "3D Object counter" plugin to check for the 1074 isotropic physical expansion of the specimen by comparing the ratio 1075 of the parasite (SAG1 staining) and the nucleus (PI staining) volumes 1076 in controlled and expanded extracellular tachyzoite samples. The 1077 nucleus sphericity index of motile, nonmotile, and intracellular 1078 tachyzoites was compared by thresholding each image object to create 1079 $3 \mathrm{D}$ binary images, whereas the "3D Object counter" plugin was next 1080 used to segment $Z$-stack binary images and retrieve the volume and 1081 surface of the 3D objects (i.e., parasite nucleus and whole body). The 1082 sphericity index was calculated using the formula where $V$ is the 1083 volume and $S$ the surface:

1084

$$
\text { sphericity } \Psi=\frac{\left(36 \pi V^{2}\right)^{1 / 3}}{S}
$$

The nucleus roundness was obtained by thresholding a Z-Max 1085 projection from the $Z$-stack images and using the "Particle analysis" 1086 plugin.

1087

For the tubulin curvature analysis in expanded specimens, we used 1088 the "Kappa" plugin in Fiji on the Z-Max projection from Z-stack 1089 images of the tachyzoite ventral cMTs (i.e., facing the substrate), 1090 which allowed tracing each MT shape and retrieving the mean 1091 curvature of each cMT as well as the curvature amplitude over the 1092 cMT length (standard deviation).

1093

MIC2 and SAG1 Analysis in Helical Trail. Extracellular 1094 tachyzoites were let to glide for 15 min prior to be fixed with PFA 1095 as described in the ExM section. A double immunolabeling was 1096 performed sequentially using the anti-TgSAG1 protein (clone TP3, 1097 $30 \mathrm{~min}, 23{ }^{\circ} \mathrm{C}$ ) and the anti-TgMIC2 (clone 6D10, overnight, $4{ }^{\circ} \mathrm{C}$ ) 1098 as primary antibodies, each followed by incubation with appropriate 1099 secondary highly cross-adsorbed antibodies $(4 \mu \mathrm{g} / \mathrm{mL}, 2 \mathrm{~h})$. Images 1100 were acquired on the Axio Imager Z1 Zeiss microscope using the 1101 Zeiss imaging software ZEN before being processed using Fiji 1102 software.

1103

To determine the size and position of the adhesion point formed at 1104 the early step of the helical cycle (i.e., coinciding with the kink) on the 1105 MIC2/SAG immunolabeled trails, we used the machine-learning- 1106 based segmentation of the real-time RICM sequence as a template. 1107 The region of interest for the trail trajectory, the angular area, and the 1108 parasite apical adhesion area were defined manually with Fiji: the 1109 distance between the center of adhesion point and the angle point was 1110 measured for six angles over a sequence of successive helical cycles, 1111 which allowed positioning the apical adhesion at about $+0.170 \mu \mathrm{m} 1112$ downstream the angle point, and the size of the adhesion point was 1113 estimated around $0.5 \mu \mathrm{m}$. Applying these values on the MIC2/SAG1 1114 labeled trails, it was possible to accurately identify the adhesion and 1115 angle area over the profiles of fluorescence intensity measured for the 1116 whole trails trajectories that in total defined 40 helical cycles 1117 performed by eight tachyzoites. The fluorescence intensity is given as 1118 a ratio by normalizing on the mean intensity value.

1119

Quantification and Statistical Analysis. Data were statistically 1120 analyzed, and plots were made using GraphPad Prism 6.0 software for 1121 Windows (La Jolla, CA, USA). Data are presented as mean \pm 1122 standard error of mean if not indicated otherwise. Figure legends 1123 include the statistical test and resulting comparison when appropriate. 1124 
1125 For Figure 4A, Figure 7A, and Figures S3A and S5B,C, an unpaired $t$ 1126 test was used with significance being represented as a $p$ value $<0.05$, 1127 and the $n$ indicated represents the parasite sample size or the number 1128 of individual microtubules analyzed. For Figure 4E, Figure 5A, and 1129 Figure S5A, an unpaired Kruskal-Wallis test was performed, and the $1130 n$ indicates the sample size. For the table in Figure 5A, a chi-square 1131 test followed by Fisher's exact text was used, with the $n$ showing the 1132 proportions analyzed.

\section{ASSOCIATED CONTENT}

\section{SI Supporting Information}

1135 The Supporting Information is available free of charge at 1136 https://pubs.acs.org/doi/10.1021/acsnano.0c01893.

1137

1138

1139

1140

1141

1142

1143

1144

1145

1146

1147

1148

1149

1150

1151

1152

1153

1154

1155

1156

1157

1158

1159

1160

1161

1162

1163

1164

1165

1166

1167

\section{AUTHOR INFORMATION}

1169 Corresponding Author

1170 Isabelle Tardieux - Institute for Advanced Biosciences (IAB),

1171 Team Membrane Dynamics of Parasite-Host Cell Interactions,

1172 CNRS UMR5309, INSERM U1209, Universite Grenoble

1173 Alpes, 38700 Grenoble, France; (1) orcid.org/0000-0002-

1174 5677-7463; Email: isabelle.tardieux@inserm.fr

\section{Authors}

1176 Georgios Pavlou - Institute for Advanced Biosciences (IAB),

1177 Team Membrane Dynamics of Parasite-Host Cell Interactions,

1178 CNRS UMR5309, INSERM U1209, Universite Grenoble

1179 Alpes, 38700 Grenoble, France

1180 Bastien Touquet - Institute for Advanced Biosciences (IAB),

1181 Team Membrane Dynamics of Parasite-Host Cell Interactions,

1182 CNRS UMR5309, INSERM U1209, Universite Grenoble

1183 Alpes, 38700 Grenoble, France
Luis Vigetti - Institute for Advanced Biosciences (IAB), Team 1184 Membrane Dynamics of Parasite-Host Cell Interactions, CNRS 1185 UMR5309, INSERM U1209, Universite Grenoble Alpes, 1186 38700 Grenoble, France

1187

Patricia Renesto - Institute for Advanced Biosciences (IAB), 1188 Team Membrane Dynamics of Parasite-Host Cell Interactions, 1189 CNRS UMR5309, INSERM U1209, Université Grenoble 1190 Alpes, 38700 Grenoble, France; TIMC-IMAG UMR 5525 - 1191 UGA CNRS, 38700 Grenoble, France 1192

Alexandre Bougdour - Institute for Advanced Biosciences 1193 (IAB), Team Host-Pathogen Interactions \& Immunity to 1194 Infections, CNRS UMR5309, INSERM U1209, Université 1195 Grenoble Alpes, 38700 Grenoble, France 1196

Delphine Debarre - Laboratoire Interdisciplinaire de Physique, 1197 UMR CNRS, 5588, Université Grenoble Alpes, Grenoble 1198 38402, France

Martial Balland - Laboratoire Interdisciplinaire de Physique, 1200 UMR CNRS, 5588, Universite Grenoble Alpes, Grenoble 1201 38402, France

Complete contact information is available at:

https://pubs.acs.org/10.1021/acsnano.0c01893

Conceptualization, I.T., M.B, G.P; Software, M.B., D.D, G.P.; 1206 Formal Analysis, G.P., B.T., D.D, L.V.; Funding acquisition, 1207 I.T.; Investigation; G.P., B.T., I.T., V.L., D.D., P.R., A.B.; 1208 Methodology, I.T., G.P., B.T., A.B.; Project administration, 1209 I.T.; Supervision, I.T., M.B., P.R.; Validation, I.T., G.P., M.B., 1210 D.D.; Visualization, G.P., B.T., L.V.; Writing-original draft, 1211 I.T. Writing-review and editing, I.T., G.P., P.R., A.B., M.B., 1212 D.D.

1213

Funding

1214

This work has been funded by the IAB department 1215 "Prevention and Therapy of Chronic Diseases (Grenoble, 1216 France) with an internal grant. The University Grenoble-Alpes 1217 and the Fondation pour la Recherche Médicale (FRM 1218 FDT201904008408) awarded to G.P. with a 3 year Ph.D. 1219 fellowship and 6 month Ph.D. extension fellowship, respec- 1220 tively.

Notes

The authors declare no competing financial interest.

1221 1222 ACKNOWLEDGMENTS

We thank T. Boudou for help with the collagen gels, T. 1225 Andersen, V. Petrolli, and A. Ruppel for help in setting and 1226 analyzing the TFM assays. They all belong to the Laboratoire 1227 Interdisciplinaire de Physique, UMR CNRS, 5588, Université 1228 Grenoble Alpes, France. We thank C. Guilluy for the AFM 1229 assays to probe gels, M. Bogeda for help in micropatterning 1230 (IAB, Grenoble), and the Alveole team for providing the 1231 composite micropatterns. We thank the therapeutic antibodies 1232 (Tab-IP) and recombinant antibodies platform from the Curie 1233 Institute, Paris, France for providing the anti-mCherry 1234 antibodies.

(1) Scarpa, E.; Mayor, R. Collective Cell Migration in Development. 1237 J. Cell Biol. 2016, 212, 143-155.

(2) Friedl, P.; Weigelin, B. Interstitial Leukocyte Migration and 1239 Immune Function. Nat. Immunol. 2008, 9, 960-969. 1240

(3) Tschumperlin, D. J. Fibroblasts and the Ground They Walk On. 1241 Physiology 2013, 28, 380-390. 
1243 (4) Raftopoulou, M.; Hall, A. Cell Migration: Rho GTPases Lead 1244 the Way. Dev. Biol. 2004, 265, 23-32.

1245 (5) Gardel, M. L.; Schneider, I. C.; Aratyn-Schaus, Y.; Waterman, C. 1246 M. Mechanical Integration of Actin and Adhesion Dynamics in Cell 1247 Migration. Annu. Rev. Cell Dev. Biol. 2010, 26, 315-333.

1248 (6) Yamada, K. M.; Sixt, M. Mechanisms of 3D Cell Migration. Nat. 1249 Rev. Mol. Cell Biol. 2019, 20, 738-752.

1250 (7) Petrie, R. J.; Koo, H.; Yamada, K. M. Generation of 1251 Compartmentalized Pressure by a Nuclear Piston Governs Cell 1252 Motility in a 3D Matrix. Science 2014, 345, 1062-1065.

1253 (8) Copos, C. A.; Walcott, S.; del Álamo, J. C.; Bastounis, E.; 1254 Mogilner, A.; Guy, R. D. Mechanosensitive Adhesion Explains 1255 Stepping Motility in Amoeboid Cells. Biophys. J. 2017, 112, 267212562682.

1257 (9) Robert-Gangneux, F.; Darde, M.-L. Epidemiology of and 1258 Diagnostic Strategies for Toxoplasmosis. Clin. Microbiol. Rev. 2012, 1259 25, 264-296.

1260 (10) Tardieux, I.; Baum, J. Reassessing the Mechanics of Parasite 1261 Motility and Host-Cell Invasion. J. Cell Biol. 2016, 214, 507-515.

1262 (11) Håkansson, S.; Morisaki, H.; Heuser, J.; Sibley, L. D. Time1263 Lapse Video Microscopy of Gliding Motility in Toxoplasma Gondii 1264 Reveals a Novel, Biphasic Mechanism of Cell Locomotion. Mol. Biol. 1265 Cell 1999, 10, 3539-3547.

1266 (12) Amino, R.; Thiberge, S.; Martin, B.; Celli, S.; Shorte, S.; 1267 Frischknecht, F.; Ménard, R. Quantitative Imaging of Plasmodium 1268 Transmission from Mosquito to Mammal. Nat. Med. 2006, 12, 2201269224.

1270 (13) Münter, S.; Sabass, B.; Selhuber-Unkel, C.; Kudryashev, M.; 1271 Hegge, S.; Engel, U.; Spatz, J. P.; Matuschewski, K.; Schwarz, U. S.; 1272 Frischknecht, F. Plasmodium Sporozoite Motility Is Modulated by the 1273 Turnover of Discrete Adhesion Sites. Cell Host Microbe 2009, 6, 5511274562.

1275 (14) Frischknecht, F.; Matuschewski, K. Plasmodium Sporozoite 1276 Biology. Cold Spring Harbor Perspect. Med. 2017, 7, a025478.

1277 (15) Amino, R.; Giovannini, D.; Thiberge, S.; Gueirard, P.; Boisson, 1278 B.; Dubremetz, J.-F.; Prévost, M.-C.; Ishino, T.; Yuda, M.; Ménard, R. 1279 Host Cell Traversal Is Important for Progression of the Malaria 1280 Parasite through the Dermis to the Liver. Cell Host Microbe 2008, 3, $128188-96$.

1282 (16) Kan, A.; Tan, Y.-H.; Angrisano, F.; Hanssen, E.; Rogers, K. L.; 1283 Whitehead, L.; Mollard, V. P.; Cozijnsen, A.; Delves, M. J.; Crawford, 1284 S.; Sinden, R. E.; McFadden, G. I.; Leckie, C.; Bailey, J.; Baum, J. 1285 Quantitative Analysis of Plasmodium Ookinete Motion in Three 1286 Dimensions Suggests a Critical Role for Cell Shape in the 1287 Biomechanics of Malaria Parasite Gliding Motility. Cell. Microbiol. 1288 2014, 16, 734-750.

1289 (17) Bichet, M.; Joly, C.; Hadj Henni, A.; Guilbert, T.; Xemard, M.; 1290 Tafani, V.; Lagal, V.; Charras, G.; Tardieux, I. The Toxoplasma-Host 1291 Cell Junction Is Anchored to the Cell Cortex to Sustain Parasite 1292 Invasive Force. BMC Biol. 2014, 12, 773.

1293 (18) Frixione, E.; Mondragón, R.; Meza, I. Kinematic Analysis of 1294 Toxoplasma Gondii Motility. Cell Motil. Cytoskeleton 1996, 34, 1521295163.

1296 (19) Leung, J. M.; Rould, M. A.; Konradt, C.; Hunter, C. A.; Ward, 1297 G. E. Disruption of TgPHIL1 Alters Specific Parameters of 1298 Toxoplasma Gondii Motility Measured in a Quantitative, Three1299 Dimensional Live Motility Assay. PLoS One 2014, 9, No. e85763.

1300 (20) Carruthers, V. B.; Tomley, F. M. Receptor-Ligand Interaction 1301 and Invasion: Microneme Proteins in Apicomplexans. Subcell Biochem 1302 2008, 47, 33-45.

1303 (21) Lagal, V.; Binder, E. M.; Huynh, M.-H.; Kafsack, B. F. C.; 1304 Harris, P. K.; Diez, R.; Chen, D.; Cole, R. N.; Carruthers, V. B.; Kim, 1305 K. Toxoplasma Gondii Protease TgSUB1 Is Required for Cell Surface 1306 Processing of Micronemal Adhesive Complexes and Efficient 1307 Adhesion of Tachyzoites: TgSUB1Microneme Protein Processing. 1308 Cell. Microbiol. 2010, 12, 1792-1808.

1309 (22) Opitz, C.; Soldati, D. The Glideosome: A Dynamic Complex 1310 Powering Gliding Motion and Host Cell Invasion by Toxoplasma 1311 Gondii. Mol. Microbiol. 2002, 45, 597-604.
(23) Frénal, K.; Dubremetz, J.-F.; Lebrun, M.; Soldati-Favre, D. 1312 Gliding Motility Powers Invasion and Egress in Apicomplexa. Nat. 1313 Rev. Microbiol. 2017, 15, 645-660.

1314

(24) Andenmatten, N.; Egarter, S.; Jackson, A. J.; Jullien, N.; 1315 Herman, J.-P.; Meissner, M. Conditional Genome Engineering in 1316 Toxoplasma Gondii Uncovers Alternative Invasion Mechanisms. Nat. 1317 Methods 2013, 10, 125-127.

1318

(25) Bichet, M.; Touquet, B.; Gonzalez, V.; Florent, I.; Meissner, 1319 M.; Tardieux, I. Genetic Impairment of Parasite Myosin Motors 1320 Uncovers the Contribution of Host Cell Membrane Dynamics to 1321 Toxoplasma Invasion Forces. BMC Biol. 2016, 14, 97.

(26) Graindorge, A.; Frénal, K.; Jacot, D.; Salamun, J.; Marq, J. B.; 1323 Soldati-Favre, D. The Conoid Associated Motor MyoH Is 1324 Indispensable for Toxoplasma Gondii Entry and Exit from Host 1325 Cells. PLoS Pathog. 2016, 12, No. e1005388.

1326

(27) Morrissette, N. S.; Sibley, L. D. Cytoskeleton of Apicomplexan 1327 Parasites. Microbiol. Mol. Biol. Rev. 2002, 66, 21-38. 1328

(28) Frénal, K.; Polonais, V.; Marq, J.-B.; Stratmann, R.; Limenitakis, 1329 J.; Soldati-Favre, D. Functional Dissection of the Apicomplexan 1330 Glideosome Molecular Architecture. Cell Host Microbe 2010, 8, 343- 1331 357.

(29) Mueller, C.; Graindorge, A.; Soldati-Favre, D. Functions of 1333 Myosin Motors Tailored for Parasitism. Curr. Opin. Microbiol. 2017, 1334 $40,113-122$.

1335

(30) Liu, J.; He, Y.; Benmerzouga, I.; Sullivan, W. J.; Morrissette, N. 1336 S.; Murray, J. M.; Hu, K. An Ensemble of Specifically Targeted 1337 Proteins Stabilizes Cortical Microtubules in the Human Parasite 1338 Toxoplasma Gondii. Mol. Biol. Cell 2016, 27, 549-571. 1339

(31) Quadt, K. A.; Streichfuss, M.; Moreau, C. A.; Spatz, J. P.; 1340 Frischknecht, F. Coupling of Retrograde Flow to Force Production 1341 During Malaria Parasite Migration. ACS Nano 2016, 10, 2091-2102. 1342

(32) Whitelaw, J. A.; Latorre-Barragan, F.; Gras, S.; Pall, G. S.; 1343 Leung, J. M.; Heaslip, A.; Egarter, S.; Andenmatten, N.; Nelson, S. R.; 1344 Warshaw, D. M.; Ward, G. E.; Meissner, M. Surface Attachment, 1345 Promoted by the Actomyosin System of Toxoplasma Gondii Is 1346 Important for Efficient Gliding Motility and Invasion. BMC Biol. 1347 $2017,15,1$.

1348

(33) Tosetti, N.; Dos Santos Pacheco, N.; Soldati-Favre, D.; Jacot, 1349 D. Three F-Actin Assembly Centers Regulate Organelle Inheritance, 1350 Cell-Cell Communication and Motility in Toxoplasma gondii. eLife 1351 2019, 8, e42669.

1352

(34) Mouw, J. K.; Ou, G.; Weaver, V. M. Extracellular Matrix 1353 Assembly: A Multiscale Deconstruction. Nat. Rev. Mol. Cell Biol. 2014, 1354 $15,771-785$.

1355

(35) Aper, S. J. A.; van Spreeuwel, A. C. C.; van Turnhout, M. C.; 1356 van der Linden, A. J.; Pieters, P. A.; van der Zon, N. L. L.; de la 1357 Rambelje, S. L.; Bouten, C. V. C.; Merkx, M. Colorful Protein-Based 1358 Fluorescent Probes for Collagen Imaging. PLoS One 2014, 9, 1359 No. e114983.

1360

(36) Ejigiri, I.; Ragheb, D. R. T.; Pino, P.; Coppi, A.; Bennett, B. L.; 1361 Soldati-Favre, D.; Sinnis, P. Shedding of TRAP by a Rhomboid 1362 Protease from the Malaria Sporozoite Surface Is Essential for Gliding 1363 Motility and Sporozoite Infectivity. PLoS Pathog. 2012, 8, 1364 No. e1002725.

1365

(37) Huynh, M.-H.; Carruthers, V. B. Toxoplasma MIC2 Is a Major 1366 Determinant of Invasion and Virulence. PLoS Pathog. 2006, 2, 1367 No. e84.

1368

(38) Gras, S.; Jackson, A.; Woods, S.; Pall, G.; Whitelaw, J.; Leung, J. 1369 M.; Ward, G. E.; Roberts, C. W.; Meissner, M. Parasites Lacking the 1370 Micronemal Protein MIC2 Are Deficient in Surface Attachment and 1371 Host Cell Egress, but Remain Virulent In Vivo. Wellcome Open Res. 1372 2017, 2, 32 .

1373

(39) Buguliskis, J. S.; Brossier, F.; Shuman, J.; Sibley, L. D. 1374 Rhomboid 4 (ROM4) Affects the Processing of Surface Adhesins and 1375 Facilitates Host Cell Invasion by Toxoplasma Gondii. PLoS Pathog. 1376 2010, 6, No. e1000858.

(40) Martiel, J.-L.; Leal, A.; Kurzawa, L.; Balland, M.; Wang, I.; 1378 Vignaud, T.; Tseng, Q.; Théry, M. Measurement of Cell Traction 1379 Forces with ImageJ. Methods Cell Biol. 2015, 125, 269-287. 
1381 (41) Hegge, S.; Uhrig, K.; Streichfuss, M.; Kynast-Wolf, G.; 1382 Matuschewski, K.; Spatz, J. P.; Frischknecht, F. Direct Manipulation 1383 of Malaria Parasites with Optical Tweezers Reveals Distinct Functions 1384 of Plasmodium Surface Proteins. ACS Nano 2012, 6, 4648-4662.

1385 (42) Stadler, R. V.; White, L. A.; Hu, K.; Helmke, B. P.; Guilford, W. $1386 \mathrm{H}$. Direct Measurement of Cortical Force Generation and Polar1387 ization in a Living Parasite. Mol. Biol. Cell 2017, 28, 1912-1923.

1388 (43) Biais, N.; Higashi, D. L.; Brujic, J.; So, M.; Sheetz, M. P. Force1389 Dependent Polymorphism in Type IV Pili Reveals Hidden Epitopes. 1390 Proc. Natl. Acad. Sci. U. S. A. 2010, 107, 11358-11363.

1391 (44) Perschmann, N.; Hellmann, J. K.; Frischknecht, F.; Spatz, J. P. 1392 Induction of Malaria Parasite Migration by Synthetically Tunable 1393 Microenvironments. Nano Lett. 2011, 11, 4468-4474.

1394 (45) Asano, S. M.; Gao, R.; Wassie, A. T.; Tillberg, P. W.; Chen, F.; 1395 Boyden, E. S. Expansion Microscopy: Protocols for Imaging Proteins 1396 and RNA in Cells and Tissues. Curr. Protoc. Cell Biol. 2018, 80, 1397 No. e56.

1398 (46) Egarter, S.; Andenmatten, N.; Jackson, A. J.; Whitelaw, J. A.; 1399 Pall, G.; Black, J. A.; Ferguson, D. J. P.; Tardieux, I.; Mogilner, A.; 1400 Meissner, M. The Toxoplasma Acto-MyoA Motor Complex Is 1401 Important but Not Essential for Gliding Motility and Host Cell 1402 Invasion. PLoS One 2014, 9, No. e91819.

1403 (47) Bubb, M. R.; Spector, I.; Beyer, B. B.; Fosen, K. M. Effects of 1404 Jasplakinolide on the Kinetics of Actin Polymerization. An 1405 Explanation for Certain In Vivo Observations. J. Biol. Chem. 2000, 1406 275, 5163-5170.

1407 (48) Sampath, P.; Pollard, T. D. Effects of Cytochalasin, Phalloidin, 1408 and PH on the Elongation of Actin Filaments. Biochemistry 1991, 30, 1409 1973-1980.

1410 (49) Periz, J.; Whitelaw, J.; Harding, C.; Gras, S.; Del Rosario 1411 Minina, M. I.; Latorre-Barragan, F.; Lemgruber, L.; Reimer, M. A.; 1412 Insall, R.; Heaslip, A.; Meissner, M. Toxoplasma Gondii F-Actin Forms 1413 an Extensive Filamentous Network Required for Material Exchange 1414 and Parasite Maturation. eLife 2017, 6, e24119.

1415 (50) Shaw, M. K.; Tilney, L. G. Induction of an Acrosomal Process 1416 in Toxoplasma Gondii: Visualization of Actin Filaments in a Protozoan 1417 Parasite. Proc. Natl. Acad. Sci. U. S. A. 1999, 96, 9095-9099.

1418 (51) Del Rosario, M.; Periz, J.; Pavlou, G.; Lyth, O.; Latorre1419 Barragan, F.; Das, S.; Pall, G. S.; Stortz, J. F.; Lemgruber, L.; 1420 Whitelaw, J. A.; Baum, J.; Tardieux, I.; Meissner, M. Apicomplexan F1421 Actin Is Required for Efficient Nuclear Entry during Host Cell 1422 Invasion. EMBO Rep. 2019, 20, e48896.

1423 (52) Gambarotto, D.; Zwettler, F. U.; Le Guennec, M.; Schmidt1424 Cernohorska, M.; Fortun, D.; Borgers, S.; Heine, J.; Schloetel, J.-G.; 1425 Reuss, M.; Unser, M.; Boyden, E. S.; Sauer, M.; Hamel, V.; Guichard, 1426 P. Imaging Cellular Ultrastructures Using Expansion Microscopy (U1427 ExM). Nat. Methods 2019, 16, 71-74.

1428 (53) Plessmann, U.; Reiter-Owona, I.; Lechtreck, K.-F. Posttransla1429 tional Modifications of $\alpha$-Tubulin of Toxoplasma Gondii. Parasitol. 1430 Res. 2004, 94, 386-389.

1431 (54) Brangwynne, C. P.; MacKintosh, F. C.; Kumar, S.; Geisse, N. 1432 A.; Talbot, J.; Mahadevan, L.; Parker, K. K.; Ingber, D. E.; Weitz, D. 1433 A. Microtubules Can. Bear Enhanced Compressive Loads in Living 1434 Cells Because of Lateral Reinforcement. J. Cell Biol. 2006, 173, 7331435741.

1436 (55) Kabir, A. Md. R.; Inoue, D.; Afrin, T.; Mayama, H.; Sada, K.; 1437 Kakugo, A. Buckling of Microtubules on a 2D Elastic Medium. Sci. 1438 Rep. 2015, 5, 17222.

1439 (56) Leung, J. M.; He, Y.; Zhang, F.; Hwang, Y.-C.; Nagayasu, E.; 1440 Liu, J.; Murray, J. M.; Hu, K. Stability and Function of a Putative 1441 Microtubule-Organizing Center in the Human Parasite Toxoplasma 1442 Gondii. Mol. Biol. Cell 2017, 28, 1361-1378.

1443 (57) Harding, C. R.; Gow, M.; Kang, J. H.; Shortt, E.; Manalis, S. R.; 1444 Meissner, M.; Lourido, S. Alveolar Proteins Stabilize Cortical 1445 Microtubules in Toxoplasma Gondii. Nat. Commun. 2019, 10, 401. 1446 (58) Cramer, L. P. Forming the Cell Rear First: Breaking Cell 1447 Symmetry to Trigger Directed Cell Migration. Nat. Cell Biol. 2010, $144812,628-632$.
(59) Huynh, M.-H.; Carruthers, V. B. Tagging of Endogenous Genes 1449 in a Toxoplasma Gondii Strain Lacking Ku80. Eukaryotic Cell 2009, 8, 1450 $530-539$.

1451

(60) Andenmatten, N.; Egarter, S.; Jackson, A. J.; Jullien, N.; 1452 Herman, J.-P.; Meissner, M. Conditional Genome Engineering in 1453 Toxoplasma Gondii Uncovers Alternative Invasion Mechanisms. Nat. 1454 Methods 2013, 10, 125-127.

1455

(61) Farhat, D. C.; Swale, C.; Dard, C.; Cannella, D.; Ortet, P.; 1456 Barakat, M.; Sindikubwabo, F.; Belmudes, L.; De Bock, P.-J.; Couté, 1457 Y.; Bougdour, A.; Hakimi, M.-A. A MORC-Driven Transcriptional 1458 Switch Controls Toxoplasma Developmental Trajectories and Sexual 1459 Commitment. Nat. Microbiol. 2020, 5, 570-583.

1460

(62) Pavlou, G.; Tardieux, I. Phenotyping Toxoplasma Invasive Skills 1461 by Fast Live Cell Imaging. Methods Mol. Biol. 2020, 2071, 209-220. 1462

(63) Davies, H. S.; Baranova, N. S.; El Amri, N.; Coche-Guérente, 1463 L.; Verdier, C.; Bureau, L.; Richter, R. P.; Débarre, D. An Integrated 1464 Assay to Probe Endothelial Glycocalyx-Blood Cell Interactions under 1465 Flow in Mechanically and Biochemically Well-Defined Environments. 1466 Matrix Biol. 2019, 78-79, 47-59.

1467

(64) Arganda-Carreras, I.; Kaynig, V.; Rueden, C.; Eliceiri, K. W.; 1468 Schindelin, J.; Cardona, A.; Sebastian Seung, H. Trainable Weka 1469 Segmentation: A Machine Learning Tool for Microscopy Pixel 1470 Classification. Bioinformatics 2017, 33, 2424-2426.

(65) Martiel, J.-L.; Leal, A.; Kurzawa, L.; Balland, M.; Wang, I.; 1472 Vignaud, T.; Tseng, Q.; Théry, M. Measurement of Cell Traction 1473 Forces with ImageJ. Methods Cell Biol. 2015, 125, 269-287. 1474 (66) Moudy, R.; Manning, T. J.; Beckers, C. J. The Loss of 1475 Cytoplasmic Potassium upon Host Cell Breakdown Triggers Egress of 1476 Toxoplasma Gondii. J. Biol. Chem. 2001, 276, 41492-41501. 1477 